\title{
Host galaxy properties and environment of obscured and unobscured $X$-ray selected active galactic nuclei in the COSMOS survey
}

\author{
C. Bornancini ${ }^{1,2 \star}$ and D. García Lambasi ${ }^{1,2 \star}$ \\ ${ }^{1}$ Instituto de Astronomía Teórica y Experimental, (IATE, CONICET-UNC), Córdoba, Argentina \\ ${ }^{2}$ Observatorio Astronómico, Universidad Nacional de Córdoba, Laprida 854, X5000BGR, Córdoba, Argentina
}

Accepted 2020 March 11. Received 2020 March 10; in original form 2019 November 8

\begin{abstract}
We analyse different photometric and spectroscopic properties of active galactic nuclei (AGNs) and quasars (QSOs) selected by their mid-IR power-law and X-ray emission from the COSMOS survey. We use a set of star-forming galaxies as a control sample to compare with the results. We have considered samples of obscured $(H R>-0.2)$ and unobscured $(H R<-0.2)$ sources including AGNs with $L_{X}<10^{44} \mathrm{erg} \mathrm{s}^{-1}$, as well as QSOs $\left(L_{X}>10^{44} \mathrm{erg} \mathrm{s}^{-1}\right)$ with 1.4 $\leq z \leq 2.5$. We also study the typical environment of these samples, by assessing neighbouring galaxy number density and neighbour properties such as colour, stellar mass, and star formation rate. We find that the UV/optical and mid-infrared colour distribution of the different AGN types differ significantly. Also, we obtain most of AGNs and QSOs to be more compact when compared to the sample of SF galaxies. In general we find that the stellar mass distribution of the different AGN sample are similar, obtaining only a difference of $\Delta \overline{\log M}=0.3 \operatorname{dex}\left(\mathrm{M}_{\odot}\right)$ between unobscured and obscured QSOs. Obscured and unobscured AGNs and QSOs reside in different local environment at small $\left(r_{p}<100 \mathrm{kpc}\right)$ scales. Our results support previous findings where AGN type correlates with environment. These differences and those found in AGN host properties cast out the simplest unified model in which obscuration is purely an orientation effect.
\end{abstract}

Key words: galaxies: active-galaxies: structure-infrared: galaxies.

\section{INTRODUCTION}

Active galactic nuclei (AGN) are compact cores of some galaxies that emit a high-luminosity and a non-stellar radiation origin. The study of AGN offers a great opportunity to know about the nature and evolution of galaxies (Miley \& De Breuck 2008; Hickox et al. 2009; Caplar, Lilly \& Trakhtenbrot 2015; Wang et al. 2017; Hickox \& Alexander 2018; Caplar, Lilly \& Trakhtenbrot 2018). It is well known and accepted the model where AGNs are closely related to the presence of a supermassive black hole (SMBH) that accretes the surrounding matter (Zel'dovich \& Novikov 1964; Salpeter 1964; Lynden-Bell 1969; Shakura \& Sunyaev 1973; Rees 1984). After the discovery of quasars [QSOs, Schmidt (1963)], we have learned a lot about the nature of AGNs (Miley \& De Breuck 2008; D’Onofrio, Marziani \& Sulentic 2012). After these first studies, it was thought that AGNs were a rare and a particular case. Today we know, after more than $50 \mathrm{yr}$ of research, that, possibly all galaxies are thought to undergo active phases where the central SMBH accretes galactic material (Volonteri, Haardt \& Madau 2003; Cattaneo et al. 2005;

\footnotetext{
^E-mail: bornancini@gmail.com (CB); diego.garcia.lambas@unc.edu.ar
} (DGL)
Springel, Di Matteo \& Hernquist 2005a; Springel et al. 2005b; Hopkins et al. 2006a,b, 2008).

There is also evidence that links some galaxy properties with their presence of a SMBH in their centres. For example, there is an amount of observational data that relates black hole and galaxy bulge mass (Kormendy 1988a,b; Magorrian et al. 1998; Marconi \& Hunt 2003; Greene, Ho \& Barth 2008; Schulze \& Wisotzki 2011; Sani et al. 2011; Beifiori et al. 2012; Kormendy \& Ho 2013; Sanghvi et al. 2014; Savorgnan et al. 2016; Yang et al. 2019; Shankar et al. 2019) or velocity dispersion (Ferrarese \& Merritt 2000; Gebhardt et al. 2000; Tremaine et al. 2002; Shields et al. 2003; Woo et al. 2006; Treu et al. 2007; Shen et al. 2008; Graham et al. 2011; Bennert et al. 2015; Shen et al. 2015; Saglia et al. 2016).

According to their optical spectra, certain AGN types are classified according to the presence of broad emission lines (FWHM $>2000 \mathrm{~km} \mathrm{~s}^{-1}$, Broad Line AGNs or Type 1) and narrow emission lines or by the absence of broad permitted emission lines (Narrow Line AGNs or Type 2). In the 1990s, a unified model was developed to explain the different observational AGNs features according to the presence of broad or narrow spectral lines (Antonucci 1993; Urry \& Padovani 1995; Netzer 2015). The unified model explains the wide variety of AGN features in terms of the anisotropic geometry of the black hole's immediate surroundings. In this model, 
AGNs have a central SMBH surrounded by a gaseous accretion disk. The broad lines observed in the spectrum would originate in high-speed gas clouds near the $\mathrm{SMBH}$, known as the 'broad line region'. Beyond this, there would be a torus-shaped structure with sizes of a few parsecs, formed by an optically thick clouds of dust and molecular gas. Further away, there would be a structure formed by low-density clouds, with dimensions of 100-300 pc that move at low speeds producing narrow lines observed in some spectra (known as 'narrow line region'). In this model the observed differences between Type 1 and Type 2 objects are due to orientation effects with respect to the line of sight to the observer. If the torus is face on, it is possible to detect the broad-line region directly and the galaxies are identified as Type 1 AGN, otherwise, AGN observed through a torus will be classified as Type 2 AGN.

Originally, this model emerged to explain the differences observed in Seyfert galaxies, that represent a particular case of lowluminosity and low-redshift AGNs (Antonucci 1993). Other unified models include also other types of active galaxies, such as radio galaxies (Urry \& Padovani 1995; Kembhavi \& Narlikar 1999). But it is well known that the powerful radio galaxies are a particular case and they are commonly identified with massive elliptical galaxies at low (Best et al. 2007; Bornancini et al. 2010) redshifts and with massive galaxy systems (such as protoclusters) at high redshifts (Venemans et al. 2002; Miley et al. 2004, 2006; Hatch et al. 2011; Overzier 2016; Shimakawa et al. 2018).

There are also other works that attempt some changes to the model. Goulding et al. (2012) analysed a sample of local heavily obscured AGNs, i.e. Compton-thick sources with $N_{H}>1.5 \times 10^{24}$ $\mathrm{cm}^{-2}$, and proposed that the dominant contribution to the observed mid-IR dust extinction is dust located in the host galaxy at much larger scales than predicted for a torus. These ideas are shared by other authors (Malkan, Gorjian \& Tam 1998; Matt 2000; Guainazzi et al. 2001; Reunanen, Kotilainen \& Prieto 2003; Goulding \& Alexander 2009), while other authors propose different geometries for the dusty absorber (Nenkova, Ivezić \& Elitzur 2002; Elitzur \& Shlosman 2006; Alonso-Herrero et al. 2011; Ramos Almeida, et al. 2011; Audibert et al. 2017).

If the unified model is correct, that is, the differences observed in the spectra are due only to orientation effects, one would expect no differences in host galaxy properties and they would have expected to have similar local galaxy environment, but the results obtained at low and high redshifts are contradictory. For a sample of lowredshift AGNs, Jiang et al. (2016) found differences in halo scale environments between different AGN types. These authors studied the clustering properties of Type 1 and Type 2 AGNs selected from the Sloan Digital Sky Survey (SDSS), matched in redshift, $\mathrm{M}_{r}$, and $L_{\text {OIII }}$, and found similar environments on larger scales. But at scales smaller than $100 \mathrm{~h}^{-1} \mathrm{kpc}$, Type 2 AGNs have significantly more neighbours than Type 1 AGNs. These authors concluded that both AGNs samples are hosted by haloes of similar masses, but Type 2 AGNs have more satellites around them.

Using a sample of low-redshift AGNs taken from the SDSS, Xu et al. (2012) calculated the spatial correlation function of galaxies with Narrow Line Seyfert 1 (NLS1) and Broad Line Seyfert 1 (BLS1) AGNs. These authors found no significant difference at scales from a few tens of kpc to a few tens of Mpc.

Allevato et al. (2014) investigated the clustering properties of a sample of $z \sim 3$ Type 1 and 2 X-ray selected AGNs. Their results show that unobscured (Type 1) AGNs inhabit higher-mass haloes compared to the obscured population (Type 2). They inferred that unobscured AGN reside in 10 times more massive haloes compared to obscured AGN. Other works also find differences in the dark matter halo masses of different AGN types. Hickox et al. (2011) present the first measurement of the clustering of mid-IR selected obscured and unobscured QSOs selected from the $9 \mathrm{deg}^{2}$ Boötes multi-wavelength survey with redshifts in the range $0.7<$ $<1.8$. These authors found that unobscured and obscured AGNs are located in dark matter halo masses of $\log \left(M_{\text {halo }}\left[\mathrm{h}^{-1} \mathrm{M} \odot\right]\right)=$ 12.7 and 13.3, respectively. Similar results were found by Donoso et al. (2014) and DiPompeo et al. (2014) for a large sample of AGNs selected from their mid-IR colour using data from the SDSS and the Wide-field Infrared Survey Explorer (WISE) catalogues. Donoso et al. (2014) found that obscured and unobscured AGNs inhabit environments with a typical dark matter halo mass of $\log \left(M_{h} / \mathrm{M}_{\odot}\right.$ $\left.h^{-1}\right) \sim 13.5$ and $\log \left(M_{h} / \mathrm{M}_{\odot} h^{-1}\right) \sim 12.4$, respectively. In a similar way, DiPompeo et al. (2014) using a very similar galaxy sample to that of Donoso et al. (2014), but applying a robust and conservative mask to WISE-selected AGNs, found that obscured QSOs reside in haloes of higher mass: $\log \left(M_{h} / \mathrm{M}_{\odot} h^{-1}\right) \sim 13.3$, while unobscured quasar host have $\log \left(M_{h} / \mathrm{M}_{\odot} h^{-1}\right) \sim 12.8$.

In the last years, based on data observations and other results obtained from numerical simulations, it has taken great relevance evolutionary models that predict the existence of stages of formation of galaxies where the AGN would be obscured and then another period where the AGN would be observed with the appearance of a bright QSO. One of the pioneering works in this topic was that of Sanders et al. (1988). These authors presented a model for the formation of ultraluminous infrared galaxies through the strong interaction, or merger of two molecular gas-rich spirals. It was proposed that these ultraluminous infrared galaxies represent the initial, dust-enshrouded stages of QSOs. Once the combined forces of AGN radiation pressure and supernovae explosions begin to sweep the dust of the nuclear region these objects can take the appearance of optical QSOs. Similar results were also found in numerical simulations (Hopkins et al. 2006a; Springel et al. 2005a; Di Matteo, Springel \& Hernquist 2005)

In Bornancini, Taormina \& Lambas (2017), we carried out a study using a sample of mid-infrared AGNs selected from the Extended Chandra Deep Field - South (ECDF-S), with spectroscopic and/or photometric redshifts, in addition to information in the X-rays. In Bornancini \& García Lambas (2018), we studied host galaxy properties and environment of a sample of Type 1 and 2 AGN taken from the COSMOS2015 catalogue, within $0.3 \leq z \leq 1.1$ selected for their emission in X-rays, optical spectra and spectral energy distribution (SED) signatures. In these works we find that obscured AGNs are located in denser local galaxy environments compared to the unobscured AGN sample.

In this paper, we present an analysis of host galaxy properties and environment using an homogeneous and larger AGN sample at high redshifts selected by their mid-IR power-law emission, combining $\mathrm{X}$-ray, optical, and spectroscopic observations. We also compare our results with a sample of star-forming galaxies selected with similar properties to the AGN sample.

This paper is organized as follows: in Section 2 we present datasets and samples selection, while in Section 3 we investigate about the properties of obscured and unobscured AGN and QSO host galaxies. The environment of the different AGN and QSO types is analysed in Section 4. In Section 5 we analyse the properties of neighbouring galaxies in the field of AGN and QSO samples. Finally, the summary and conclusions of our work are presented in Section 6. Throughout the work we will use the AB magnitude system (Oke \& Gunn 1983) and we will assume the same $\Lambda$ CDM cosmology adopted by Laigle et al. (2016) with $\mathrm{H}_{0}=70 \mathrm{~km} \mathrm{~s}^{-1}$ $\mathrm{Mpc}^{-1}, \Omega_{M}=0.3, \Omega_{\Lambda}=0.7$. 


\section{DATASETS}

Observational data used in this paper were obtained from the Cosmic Evolution Survey [COSMOS, Scoville et al. (2007)], especially from the COSMOS2015 ${ }^{1}$ catalogue (Laigle et al. 2016). The COSMOS survey is a multi-wavelength photometric and spectroscopic survey of $2 \mathrm{deg}^{2}$, with imaging data from X-ray to radio wavelengths.

The COSMOS2015 catalogue includes GALEX NUV $(0.23 \mu \mathrm{m})$ observations (Zamojski et al. 2007), CFHT/MegaCam (u*-band) (Sanders et al. 2007), Subaru Suprime-Cam optical bands (B, V, g, r, i, $\mathrm{z}^{++}$) (Taniguchi et al. 2007, 2015). Also near-infrared YJHKsband data taken with WIRCam and Ultra-VISTA data (McCracken et al. 2010, 2012). Four Spitzer Infrared Array Camera (IRAC) bands [(3.6), (4.5), (5.8), and (8.0) $\mu \mathrm{m}$ ], S-COSMOS (Sanders et al. 2007), the Spitzer Extended Mission Deep Survey (Ashby et al. 2013), and the Spitzer S-CANDELS survey (Ashby et al. 2015). In addition, there are several $X$-ray surveys in this area: XMM-COSMOS (Hasinger et al. 2007; Cappelluti et al. 2009), CCOSMOS (Elvis et al. 2009; Civano et al. 2016), and the NuSTAR survey (Civano et al. 2015).

\subsection{AGN and SF galaxy sample selection}

The main goal of our work is to analyse the environment and properties of X-ray selected AGNs and the galaxies located in their environments. For this, we selected a sample of AGNs along with a comparison sample formed by SF galaxies with $1.4 \leq z \leq 2.5$. The sample of AGNs used in this work was selected from the Chang et al. (2017) catalogue. This catalogue is based on objects with mid-IR (MIR) $24 \mu \mathrm{m}$ emission $\left(S_{24 \mu m} \geq 80 \mu \mathrm{Jy}\right.$, Le Floc'h et al. 2009). These authors matched this sources with the COSMOS2015 catalogue (Laigle et al. 2016) in order to obtain photometry from the optical to the far-IR (FIR). According to a colour-colour selection, they have selected IR power-law AGN candidates (Lacy et al. 2004). It is well known that galaxies dominated by AGN emission typically show a power-law SED at near- and mid-IR wavelengths. In Fig. 1, it can be seen the (4.5)-(8.0) versus (3.6)-(5.8) colour-colour diagram for objects detected in $24 \mu \mathrm{m}$. The box represents the selection criteria presented by Chang et al. (2017) for AGN candidates with power-law emission with flux densities monotonically rising from 3.6 to $8 \mu \mathrm{m}$.

The derived catalogue was then cross-matched with the X-ray catalogue presented by Marchesi et al. (2016). These authors identified $1770 \mathrm{X}$-ray sources from the master spectroscopic catalogue available for the COSMOS collaboration (M. Salvato et al., in preparation).

The selection of AGNs with X-ray emission presents great advantages to those selected in the optical or in the IR. X-ray selected AGNs are less affected by obscuration and contamination from non-nuclear emission, mainly due to star formation processes, which are far less significant than in optical and infrared surveys (Donley et al. 2008, 2012; Lehmer et al. 2012; Stern et al. 2012).

In this paper, we study a sample of obscured (absorbed) and unobscured (unabsorbed) AGNs and QSOs in the X-rays.

To accomplish it, we analyse the distribution of the AGNs in the hardness ratio versus hard-X-ray luminosity diagram. The hardness ratio (HR, which is an indication of the X-ray spectral shape) of

${ }^{1}$ The catalogue can be downloaded from ftp://ftp.iap.fr/pub/ from_users/hjmcc/COSMOS2015/

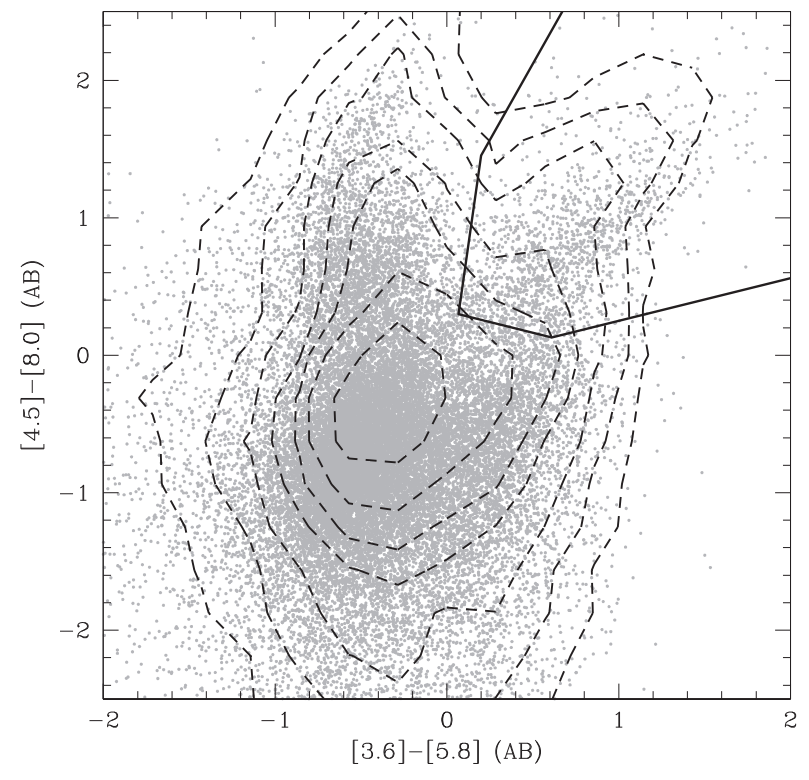

Figure 1. (4.5)-(8.0) versus (3.6)-(5.8) colour-colour diagram for objects selected in $24 \mu \mathrm{m}$. The area enclosed by the thick solid lines represents the selection criteria of Chang et al. (2017) for AGN candidates with power law emission and flux densities monotonically rising from 3.6 to $8 \mu \mathrm{m}$.

each X-ray source using the following relation,

$\mathrm{HR}=\frac{H-S}{H+S}$,

where $H$ and $S$ are the count rates in the hard $(2-10 \mathrm{keV})$ and soft (0.5-2 keV) bands, respectively.

In Fig. 2, we plot the hardness ratio as a function of hard X-ray luminosity $(2-10 \mathrm{keV})$ for a sample of AGNs with $1.4 \leq z_{\text {spec }} \leq 2.5$. The dashed horizontal line shows the HR value $(H R=-0.2)$ for a source with a neutral hydrogen column density, $N_{H}>10^{21.6} \mathrm{~cm}^{2}$ at $z>1$ (Gilli et al. 2009), which is used by several authors (Gilli et al. 2009; Treister et al. 2009; Marchesi et al. 2016) to separate obscured and unobscured sources in the X-rays. This is due to the the fact that soft X-ray emission of obscured AGNs tend to be absorbed, while hard X-ray are able to escape.

To select the AGN and QSO samples we use the selection criteria according to the luminosity in the hard X-rays, i.e. sources with hard X-ray luminosities $L_{X}<10^{44} \mathrm{erg} \mathrm{s}^{-1}$ are classified as AGNs, while sources with $L_{X} \geq 10^{44} \mathrm{erg} \mathrm{s}^{-1}$ are classified as QSOs (Treister et al. 2009).

The final AGN sample consists of 36 and 62 obscured and unobscured AGNs and 46 and 105 obscured and unobscured QSOs. In Fig. 3 we plot the spectroscopic redshift distribution for the final sample of AGNs and QSOs (obscured and unobscured, respectively). We applied a Kolmogorov-Smirnov (KS) test on the redshift distribution of both AGN and QSO samples. We find that the redshift distribution are similar at 72 per cent and 65 per cent for the sample of obscured and unobscured QSO and AGN samples.

In order to compare some properties of the AGNs sample, we have selected a sample of SF galaxies. For this reason we use two different selection criteria. First, we used a Lyman-break selection technique identified in the $N U V-B$ versus $B-V$ diagram proposed by Ly et al. (2009) to select SF galaxies with $z \approx 1.8-2.8$. In Fig. 4 , left panel, we plot $N U V-B$ versus $B-V$ colours of galaxies with $B$ $<27$, which is the $3 \sigma$ detection limit (Laigle et al. 2016). The box 


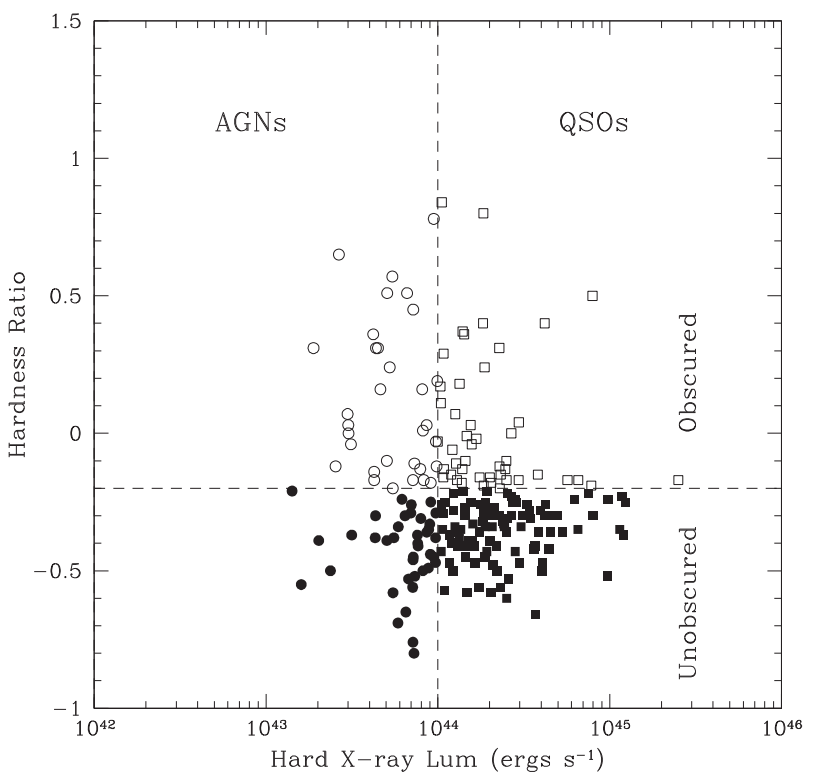

Figure 2. Hardness ratio as a function of hard (2-10 keV) X-ray luminosity. Open and filled circles and squares represent obscured and unobscured AGNs and QSOs, respectively. Vertical dashed lines show the typical separation for AGNs and QSOs used in the X-rays. The dashed horizontal line shows the HR value for a source with $N_{H}>10^{21.6} \mathrm{~cm}^{2}$ at $z>1$, which is used to separate obscured and unobscured sources in the X-rays.

represents the empirical selection criteria for the LBG sample with $z \approx 1.8-2.8$ proposed by Ly et al. (2009).

In order to avoid contamination by possible objects that are not associated with SF galaxies, we used the BzK selection technique (Daddi et al. 2004; Kong et al. 2006). This photometric technique was designed to identify star-forming (sBzK) and passive (pBzK) galaxies at $z>1.4$. In Fig. 4, right panel, we plot $z^{\prime}-K$ versus $B-z^{\prime}$ colour-colour diagram with the delimited regions occupied by star-forming and passive old galaxies (Daddi et al. 2004). We plot objects previously selected with the Lyman-break selection

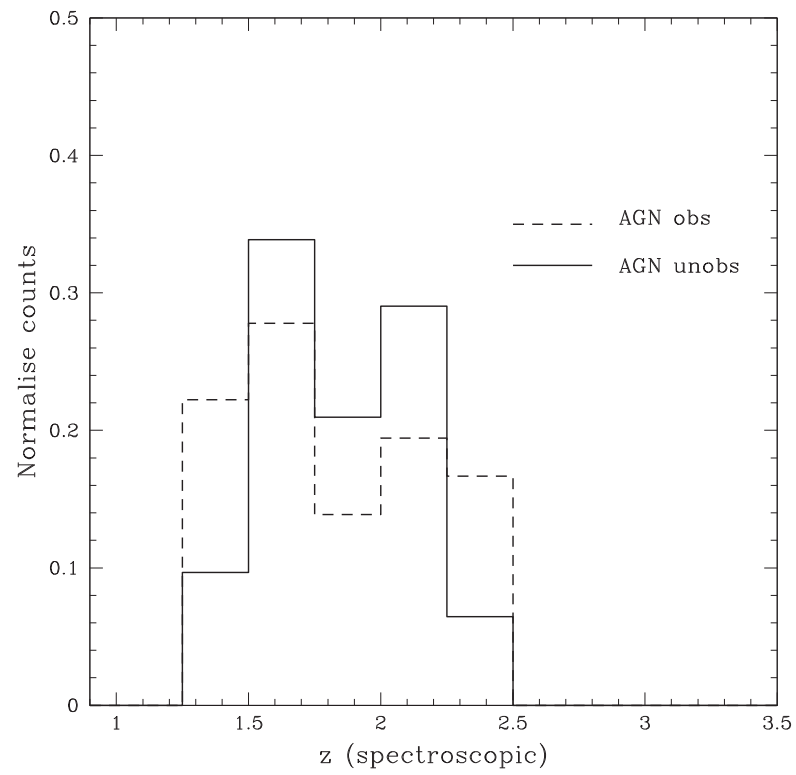

technique identified in the $N U V-B$ versus $B-V$ diagram. As it can be seen, some objects have colours outside the selection region of $\mathrm{SF}$ galaxies. For this reason, we have excluded these objects from our SF galaxy sample.

We find that only a small percentage ( $<3$ per cent) of SF galaxies have spectroscopic redshift measurements, so we decided to use only photometric redshifts. In order to select objects with good photometric redshift estimations, we chose those whose width of the 68 per cent confidence interval is less than 0.1. After this, we choose those objects with the same photometric redshift range 1.4 $\leq z_{p h} \leq 2.5$ and with $M_{K}<-22$, whose values match those found in the AGN/QSO samples. The number of SF galaxies obtained is 3157 objects.

\section{HOST GALAXY PROPERTIES}

\subsection{Mid-infrared colours}

In this section, we study the UV/optical/IR colour of the AGN and QSO samples. In Fig. 5 we plot optical-IR colour, $r-(4.5)$ versus $4.5 \mu \mathrm{m}$ for obscured and unobscured AGNs (left panel) and QSOs (right panel). In each case,, we also show the corresponding $r-(4.5)$ colour distribution. As previous works, Hickox et al. (2007), Hickox et al. (2011), Bornancini et al. (2017), Bornancini \& García Lambas (2018), we find for the AGN sample that the $r-(4.5)$ colour distribution is bimodal. For the sample of AGNs, we find that the population of obscured AGNs consists mostly of objects with predominantly red colours $[r-(4.5)>3]$, in addition to a small population of AGNs with blue colours (see Fig. 5, left panel). For unobscured AGNs, we find a similar but in reverse result, i.e. it consists mostly of objects with predominantly blue colours, in addition to a small population of AGNs with red colours (see Fig. 5, left panel). In the case of obscured QSOs (see Fig. 5, right panel), we observe two similar populations of objects with red and blue colours, while for the sample of unobscured QSOs, we find that most objects have predominantly blue colours with a peak near $r-(4.5) \sim 1.5$.

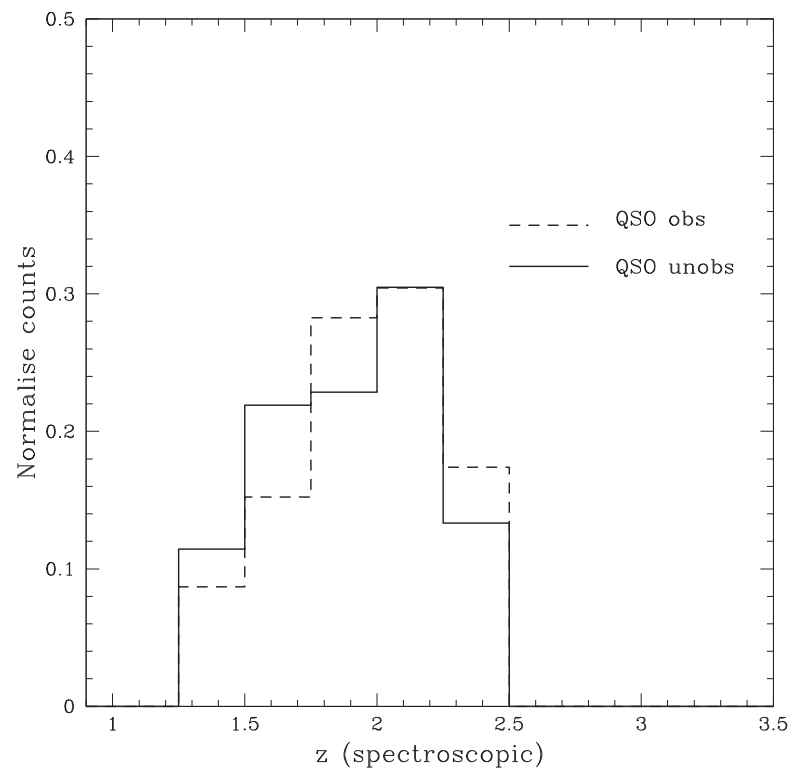

Figure 3. Normaliszed redshift distribution for the final selected obscured (dashed line histogram) and unobscured (solid line histogram) AGNs (left panel) and the corresponding distribution for QSOs (right panel). 

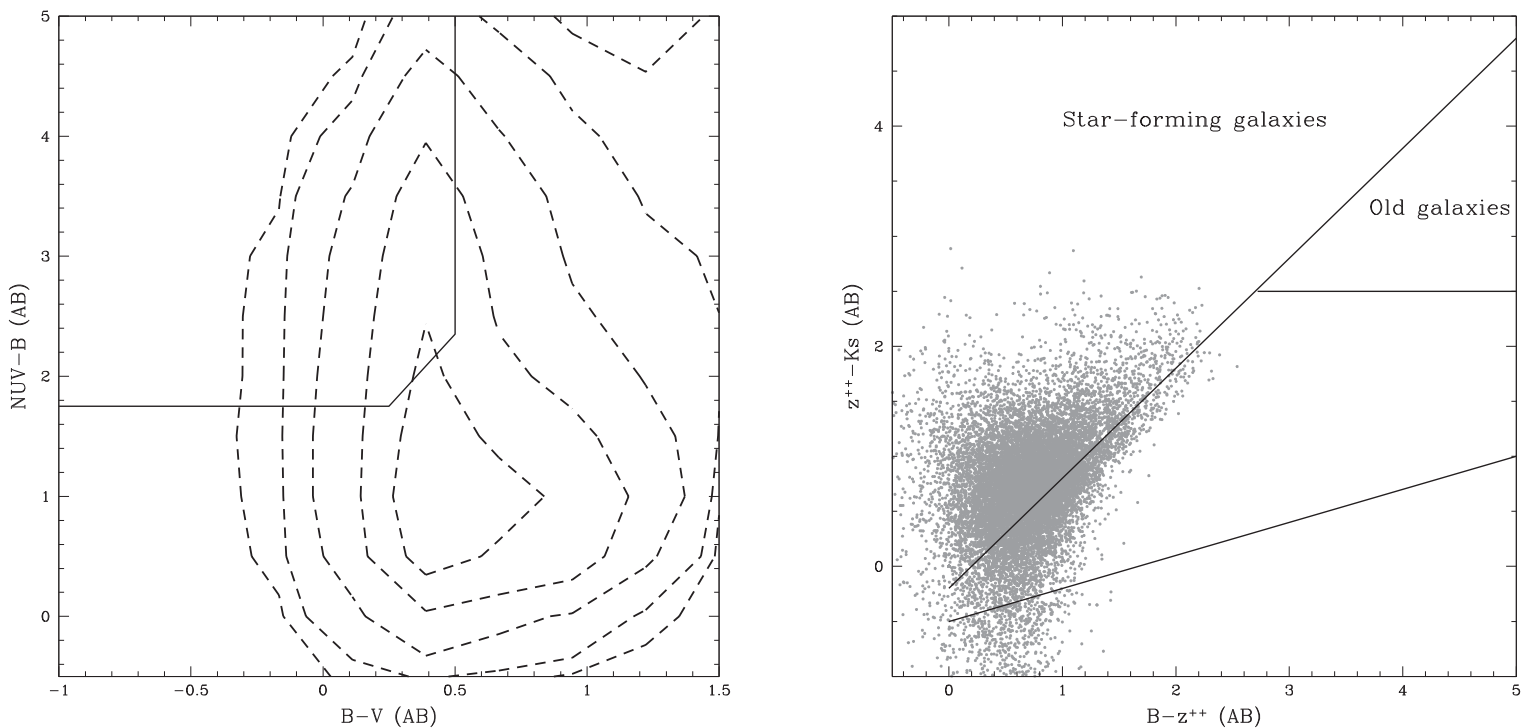

Figure 4. Left panel: $N U V-B$ versus $B-V$ colour of galaxies with $B<27$. The upper left box represents the empirical selection criteria for LBG galaxies proposed by Ly et al. (2009). Right panel: $z^{\prime}-K$ versus $B-z^{\prime}$ diagram illustrating the selection criteria for the BzK samples, showing the sBzK (star-forming galaxies) and pBzK (passive old galaxies) regions. Grey points represents objects previously selected with the Lyman-break selection technique shown in the left panel.
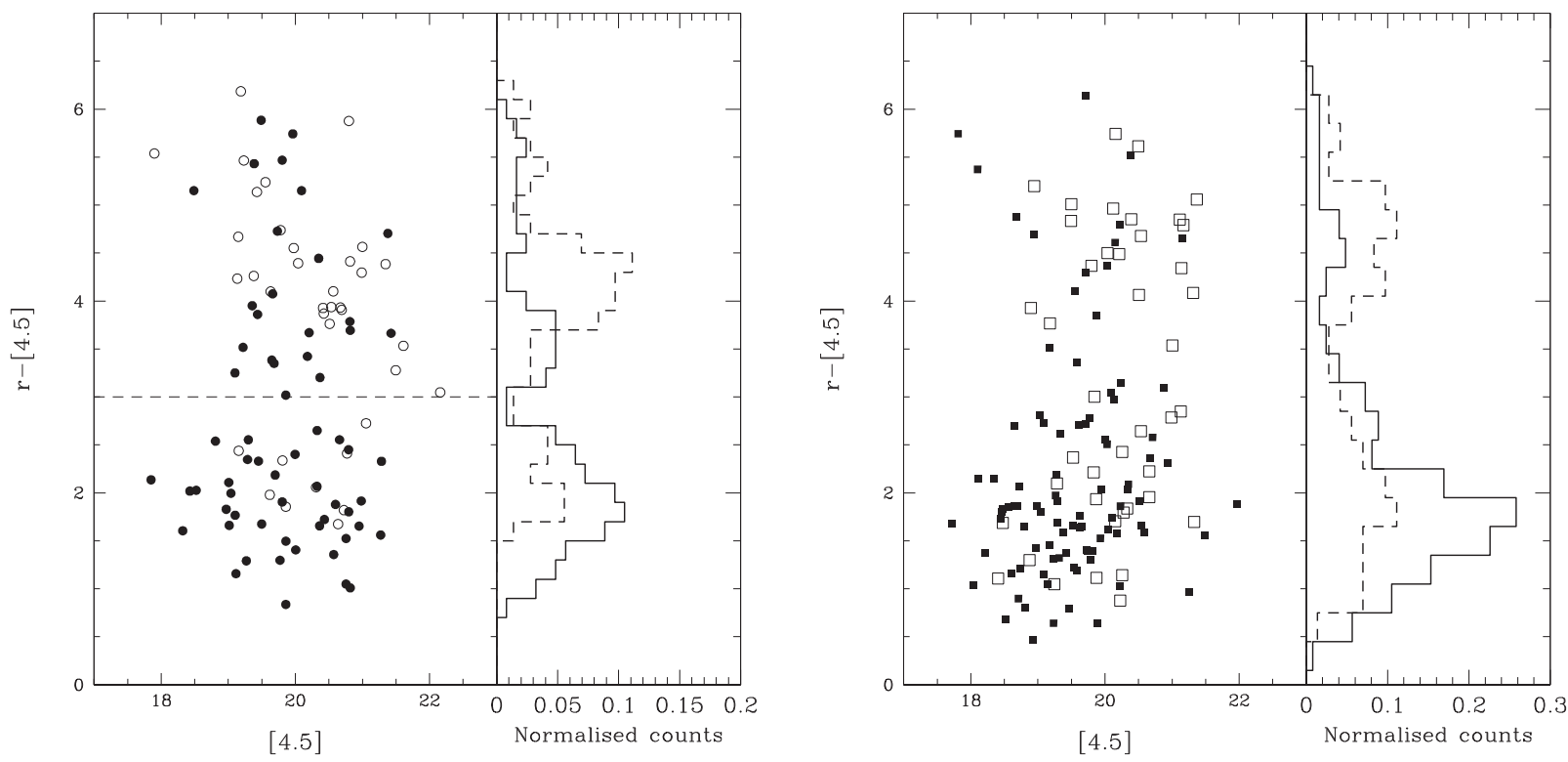

Figure 5. Left: optical-IR colour, $r-(4.5)$ versus $4.5 \mu \mathrm{m}$ for obscured (open circles) and unobscured (filled circles) AGNs. Right: the corresponding colours for obscured (open squares) and unobscured (filled squares) QSOs. Right panels in each figure show the corresponding $r-(4.5)$ colour distribution of obscured (dashed lines) and unobscured (solid line histogram) AGN and QSO samples.

In order to determine and quantify the bimodality found in the optical-IR colours, we have used the Gaussian mixture modeling (GMM) code of Muratov \& Gnedin (2010). This code performs a GMM test and uses three indicators to distinguish unimodal and bimodal distributions: the kurtosis of the distribution, the separation of the peaks $(D)$, and the probability of obtaining the same $\chi^{2}$ from a unimodal distribution $\left(p\left(\chi^{2}\right)\right)$. The separation of the peaks $(D)$ is calculated as

$D=\frac{\left|\mu_{1}-\mu_{2}\right|}{\sqrt{\frac{\sigma_{1}^{2}+\sigma_{2}^{2}}{2}}}$, where $\mu_{1}$ and $\mu_{2}$ are the mean values of the two peaks of the proposed bimodal distribution, and $\sigma_{1}, \sigma_{2}$ are the corresponding standard deviations. The distribution is considered bimodal by required negative kurtosis, $D>2$, and $p\left(\chi^{2}\right)<0.001$.

For the sample of obscured AGNs, we find kurtosis $=-0.85$, $\mu_{1}=2.1 \pm 0.3, \mu_{2}=4.4 \pm 0.2, D=3.9 \pm 0.6$, and $p\left(\chi^{2}\right)<0.001$. For unobscured AGNs, we find kurtosis $=-0.41, \mu_{1}=2.1 \pm 0.2$, $\mu_{2}=4.5 \pm 0.5, D=3.2 \pm 0.9$, and $p\left(\chi^{2}\right)<0.001$. While, for the sample of obscured QSOs, we find kurtosis $=-1.50, \mu_{1}=$ $1.9 \pm 0.2, \mu_{2}=4.7 \pm 0.1, D=4.6 \pm 0.6$, and $p\left(\chi^{2}\right)<0.001$ and for unobscured QSOs, we find kurtosis $=+0.95, \mu_{1}=1.7 \pm 0.1$, $\mu_{2}=4.1 \pm 0.6, D=3.3 \pm 1.4$, and $p\left(\chi^{2}\right)<0.001$. 

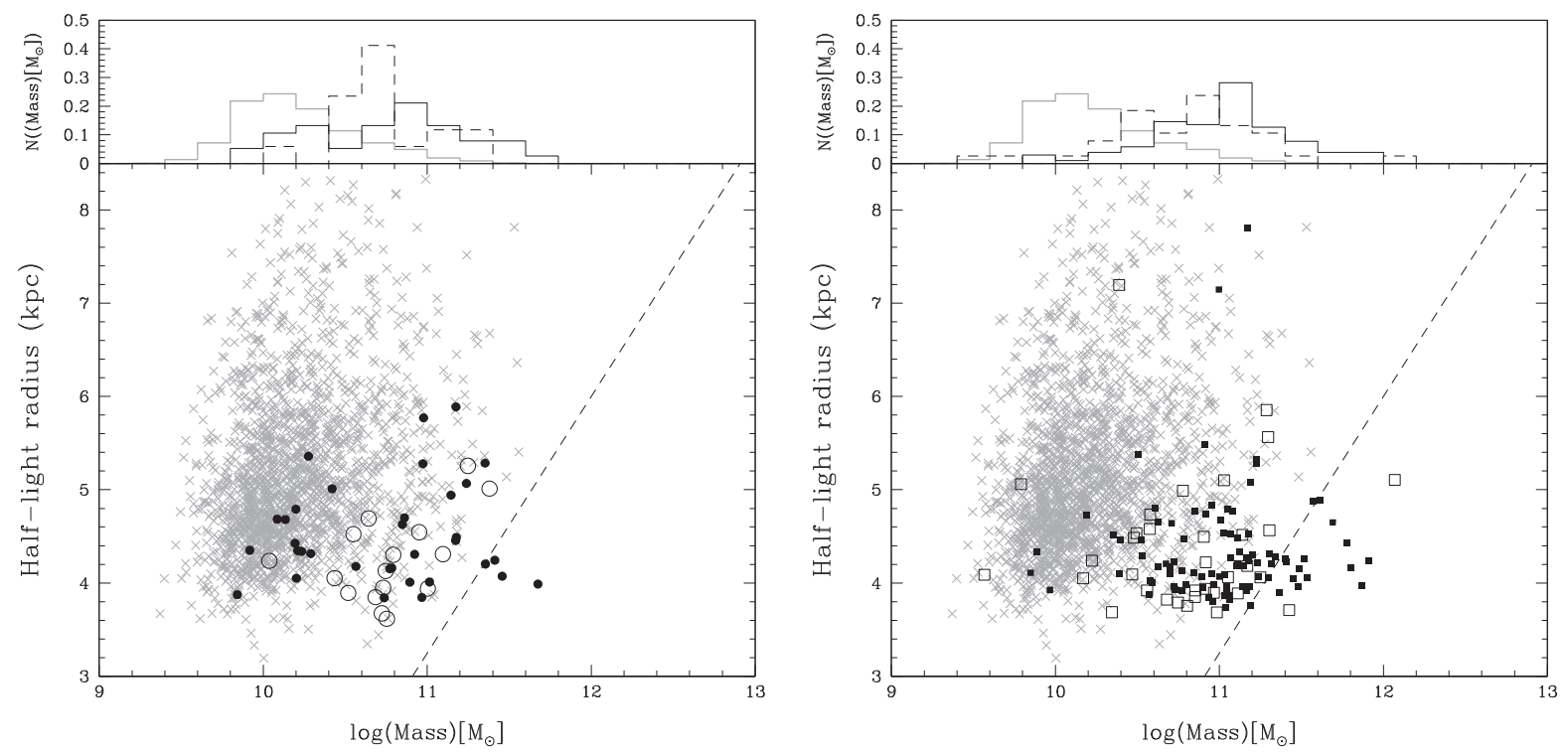

Figure 6. Stellar mass-size relation for our sample of obscured and unobscured (open and filled circles) AGNs (left panel) and for obscured and unobscured (open and filled squares) QSOs (right panel). Graey crosses represent values obtained for SF galaxies. The dashed black line represent the selection criterion for compact galaxies proposed by Barro et al. (2013). The upper panels in each figure show the stellar mass distribution for obscured and unobscured (dashed and solid lines) AGNs (left panel), obscured and unobscured (dashed and solid lines) QSOs (right panel), and SF galaxies (grey dot line).

The colour separation limit matches approximately the value found by Hickox et al. (2011) and Bornancini et al. (2017), which corresponds to $r-(4.5) \sim 3$, for the sample of obscured and unobscured AGNs and for obscured QSOs. For unobscured QSOs, we find that $r-(4.5)$ colour distribution it is not bimodal, because kurtosis is positive.

\subsection{Mass-size relation}

In this section, we analyse the mass-size relation for the AGN and QSO samples. In the local Universe, it is well known that galaxies are distributed in two large groups: one formed by passive galaxies with predominantly red colours and old stellar populations, and another group consisting of blue, star-forming galaxies (Kauffmann et al. 2003; Baldry et al. 2004). In addition, these objects have different morphologies. At a given stellar-mass, star-forming galaxies are typically larger and have disk-like morphologies, while passive ones are represented by spheroids with large concentrations of light (Williams et al. 2010; Wuyts et al. 2011).

The discovery of passive galaxies at $z \sim 2$, with small sizes, called compact quiescent galaxies or red nuggets (Damjanov et al. 2009) showed these objects to be $\sim 5$ times smaller than local, equal-mass analogues (Daddi et al. 2005; Trujillo et al. 2007; Toft et al. 2007; Cassata et al. 2007; Szomoru et al. 2011), in contrast with starforming galaxies at high redshifts identified with large disks (Kriek et al. 2009; Förster Schreiber et al. 2009).

For this reason, we cross-correlated our AGN and QSO samples with the catalogue presented in Bongiorno et al. (2012). These authors calculated colours, stellar masses and star-formation rates (SFR) of a sample of X-ray AGNs selected from the XMMCOSMOS catalogue. These parameters were carefully calculated using their SEDs, which have been parametrized using a twocomponent (AGN+galaxy) model fit. Fig. 6 shows a mass-size diagram for our sample of obscured and unobscured (open and filled circles) AGNs (left panel) and for obscured and unobscured (open and filled squares) QSOs (right panel). We also plot the corresponding values for the SF galaxy sample (grey crosses). For all samples we have used the half-light radii taken from the Laigle et al. (2016) catalogue. Dashed lines in Fig. 6 show the selection criterion for compact galaxies proposed by Barro et al. (2013), $M / r_{e}^{1.5}=10.3 \mathrm{M}_{\odot} \mathrm{kpc}^{-1.5}$. As proposed by Barro et al. (2013, 2014), the right region encloses most of the quiescent population at $\mathrm{z}>1.4$. In general, we find that most of AGNs and QSOs are more compact compared to the sample of SF galaxies. While only 12 per cent of unobscured AGNs reside in the region occupied by compact massive galaxies, we find that 15 per cent and 5 per cent of the unobscured and obscured QSOs reside in this region.

We have also included the corresponding stellar mass distribution for the samples of AGNs, QSOs, and SF galaxies. The stellar mass of the SF galaxy sample were taken from the catalogue of Laigle et al. (2016) (MASS_BEST). As it can be seen, AGNs and QSOs are identified with massive host galaxies compared to the sample of SF galaxies. The stellar mass distribution of obscured and unobscured AGNs are similar. For the obscured and unobscured AGNs we find $\overline{\log M_{o b s}}=10.7\left(\mathrm{M}_{\odot}\right), \sigma_{o b s}=0.3$, and $\overline{\log M_{\text {unobs }}}=10.8\left(\mathrm{M}_{\odot}\right)$, $\sigma_{\text {unobs }}=0.5$, respectively. For the sample of QSOs, we find a difference of $\Delta \overline{\log M}=0.3 \pm 0.1 \mathrm{dex}\left(\mathrm{M}_{\odot}\right)$, obtaining $\overline{\log M_{\text {obs }}}=10.7$ $\left(\mathrm{M}_{\odot}\right), \sigma_{\text {obs }}=0.5$, and $\overline{\log M_{\text {unobs }}}=11.0\left(\mathrm{M}_{\odot}\right), \sigma_{\text {unobs }}=0.5$. For the sample of SF galaxies we find $\overline{\log M_{S F}}=10.2\left(\mathrm{M}_{\odot}\right), \sigma_{o b s}=0.3$. This last result is in contradiction with those found by Bongiorno et al. (2012) and Bornancini \& García Lambas (2018). Bongiorno et al. (2012) found that the stellar mass distribution of obscured and unobscured AGN is nearly independent of the AGN type. Analysing a sample of Type 1 and 2 AGNs with $z<1$, Bornancini \& García Lambas (2018) found that, despite uncertainties, the mean stellar mass of Type 2 sources is 0.2 dex higher than that of Type 1 [see discussion in Zou et al. (2019)].

\subsection{UV, optical, and near-IR colours}

It is well known that galaxy colours in the near-UV (NUV), optical ( $r$-band), near-infrared ( $J$-band or $K$-band), or with similar filters can 

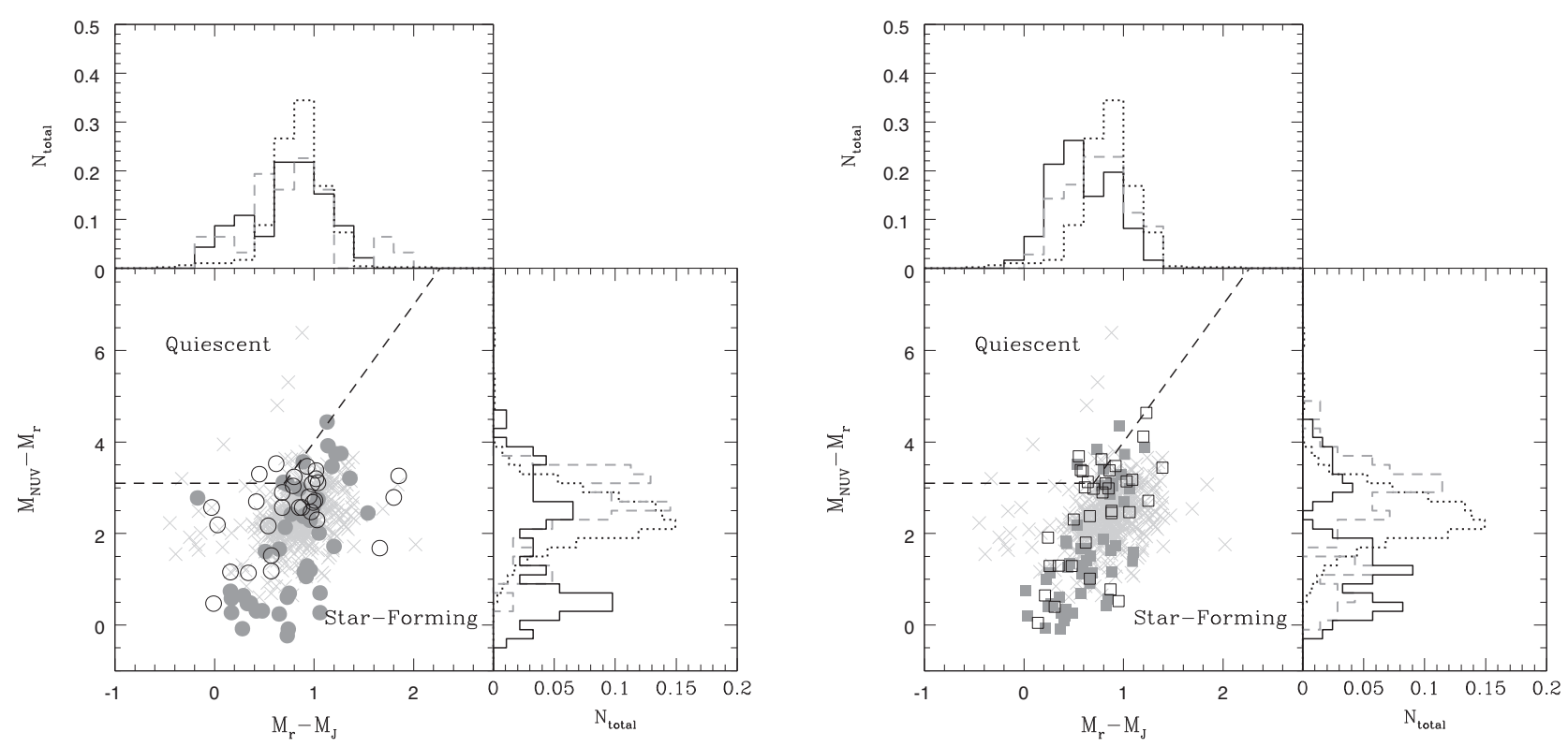

Figure 7. Rest-frame $M_{N U V}-M_{r}$ versus $M_{r}-M_{J}$ colour diagram. Grey crosses represent SF galaxies, while open and filled circles represent obscured and unobscured AGNs (left panel). Right panel: Same as left panel for the sample of obscured (open squares) and unobscured (filled squares) QSOs. Dashed lines show the limits used to empirically separate quiescent from star-forming galaxies taken from Ilbert et al. (2013). Upper and right panels show the $M_{r}-M_{J}$ and $M_{N U V}-M_{r}$ colour distribution for obscured and unobscured AGNs (dashed and solid lines) and for obscured and unobscured QSOs (dashed and solid lines). Dot histograms in upper and right panels show the corresponding colours for the sample of SF galaxies.

be used to separate star-forming from quiescent galaxies (Williams et al. 2009; Arnouts et al. 2013; Ilbert et al. 2013; Lin et al. 2016; Moutard et al. 2016). One of the first works using this colour-colour diagram was that of Williams et al. (2009). These authors found that star-forming and quiescent galaxies occupy two distinct regions in the rest-frame $(U-V)$ versus $(V-J)$ colour-colour diagram. Arnouts et al. (2013) analysing a sample of galaxies with spectroscopic redshifts between $0.2<z<1.3$ in the COSMOS survey, found a very good correlation between the specific star-formation (sSFR, which measures the SFR per unit galaxy stellar mass) and the position of these two galaxy populations in the $N U V-r$ versus $r-K$ diagram. Galaxies located in the quiescent region have low-sSFR values, such as those found in passive galaxies with little or null SFR, showing that the $N U V-r$ colour is a good tracer of the specific SFR, since the NUV band is sensitive to recent star formation and the $r$-band to old stellar populations. This method is also valid for separating galaxy morphological types. Patel et al. (2011) found that the quiescent region is dominated by galaxies with strong bulge components while the star-forming region is comprised of disks.

Fig. 7 shows the rest-frame $M_{N U V}-M_{r}$ versus $M_{r}-M_{J}$ colour diagram for obscured and unobscured (open and filled circles, respectively) AGNs (left panel) and for obscured and unobscured (open and filled squares) QSOs (right panel). The dashed lines representing the boundaries that separate regions occupied by quiescent and star-forming galaxies. Quiescent objects are those with $M_{N U V}-M_{r}>3\left(M_{r}-M_{J}\right)+1$ and $M_{N U V}-M_{r}>3.1$ while starforming galaxies occupy regions outside of this criterion (Ilbert et al. 2010, 2013). We also included the corresponding colours for SF galaxies. According to the results obtained in the Section 3.2, and with the aim at comparing host galaxies with similar stellar masses, we select SF galaxies with $\log \left(\mathrm{M}_{\odot}\right)>10.5$ (grey crosses). The final number of SF galaxies is 462 objects.

As it can be seen, the majority of the AGNs and QSOs sample falls inside the region occupied by the star-forming galaxy population.
In Bornancini \& García Lambas (2018) we conducted a similar study for a sample of Type 1 and 2 AGN with $0.3 \leq z \leq 1.1$ selected for their emission in X-rays, optical spectra, and SED signatures. In this work, we found that Type 1 AGNs are exclusively located in the region populated by star-forming galaxies in the $M_{N U V}-M_{r}$ versus $M_{r}-M_{J}$ colour diagram, whereas Type 2 are divided between the region occupied by quiescent ( 29 per cent) and star-forming galaxies (71 per cent). The majority of the AGN sample analysed in this work have hard X-ray luminosities similarly to those found in normal AGNs or Seyfert-like galaxies $\left(10^{42}<L_{X}<10^{44} \mathrm{erg} \mathrm{s}^{-1}\right)$. Only 7 per cent and 20 per cent of Type 2 and 1 objects were found to have hard X-ray luminosities similarly to those in QSOs $\left(L_{X}>\right.$ $10^{44} \mathrm{erg} \mathrm{s}^{-1}$ ).

In this work, we also find bimodalities in $M_{N U V}-M_{r}$ colour distribution in unobscured AGN and QSO samples and in obscured QSOs. The results obtained using the GMM code are as follows: for the obscured QSO sample, we find kurtosis $=-1.52, \mu_{1}=$ $0.3 \pm 0.1, \mu_{2}=3.0 \pm 0.3, D=4.6 \pm 1.0$, and $p\left(\chi^{2}\right)<0.001$. For the unobscured QSO sample, we find kurtosis $=-0.80, \mu_{1}=$ $1.0 \pm 0.1, \mu_{2}=3.3 \pm 0.2, D=4.5 \pm 0.6$, and $p\left(\chi^{2}\right)<0.001$. While for unobscured AGN, kurtosis $=-1.15, \mu_{1}=0.6 \pm 0.1$, $\mu_{2}=2.7 \pm 0.3, D=3.4 \pm 0.6$, and $p\left(\chi^{2}\right)=0.002$. In the case of obscured AGNs, the colour distribution are not bimodal and we have obtained: kurtosis $=+1.3, \mu_{1}=1.9 \pm 0.9, \mu_{2}=2.9 \pm 0.8$, $D=1.9 \pm 2.0$, and $p\left(\chi^{2}\right)=0.03$.

Unobscured AGNs and QSOs have $M_{N U V}-M_{r}$ colours slightly blue than SF galaxies. We find the following parameters for the SF galaxy sample for a unimodal distribution: kurtosis $=+4.85, \mu_{1}=$ $2.40 \pm 0.03$, and $\sigma=0.60 \pm 0.03$.

\subsection{Possible contamination by non-thermal light}

The presence of non-thermal light coming from the innermost parts of powerful QSOs can cause biases in the measurements of some host galaxy parameters, such as colours and masses. 
Pierce et al. (2010) describe the effect of AGN light on host galaxy optical $(U-B)$ and UV-optical colours $(N U V-r)$, as determined from X-ray selected AGN host galaxies at $z \sim 1$. These authors conclude that, in general, an active nucleus does not significantly affect the integrated colours of an AGN host galaxy, even when near-UV colours are used.

Hickox et al. (2009) studied a sample of AGNs with $0.25<$ $z<0.8$, selected in the radio, X-ray and IR bands from the AGN and Galaxy Evolution Survey (AGES). These authors calculated the correction for AGN contamination in the $u-r$ host galaxy colours finding that the typical correction for nuclear contamination ranges from 0 to $0.3 \mathrm{mag}$.

In a similar way Kauffmann et al. (2007), using a low-redshift sample of optically selected AGNs, found a lack of colour contamination among the AGN host galaxies in their sample.

We also carried out a visual inspection of high-resolution (0.03\%/pixel) HST images (F814W filter) of our sample of AGNs finding that only $<10$ per cent of the total AGNs + QSOs present visible nuclear point source in their optical images. ${ }^{2}$ Following the previous results, we estimate that the correction for AGN contamination on host galaxy colours is less than $\sim 0.3 \mathrm{mag}$. and so the results are not affected. The estimated maximum difference of 0.3 mag. corresponds approximately to the point size in Fig. 7.

\subsection{Morphological analysis}

In this section, we analyse the morphology of the different AGN host galaxies according to non-parametric measures, such as the Gini coefficient and the M20 parameter. The Gini coefficient is defined as the absolute value of the difference between the integrated cumulative distribution of galaxy intensities and a uniform intensity distribution (Abraham, van den Bergh \& Nair 2003), i.e. quantifies to what extent light is equally distributed among the pixels in an image, regardless of their spatial location (Lotz, Primack \& Madau 2004). High Gini coefficient values mean that most of the light is coming from just a few pixels and low values implies that all pixels are more or less equally bright.

The M20 parameter, which was introduced by Lotz et al. (2004), measure the spatial extent of the brightest galaxy regions, computed by measuring the second-order moment of the brightest 20 per cent of the galaxy pixels, defined as the sum of the intensity of each pixel multiplied by the square of the distance from the centre of the galaxy for the brightest 20 per cent of the pixels in a galaxy. It is more sensitive to the presence of bright regions at large galactocentric distances and provides information of merger signatures such as multiple nuclei, tidal tails, and bars (Lotz et al. 2004; Kong et al. 2009; Wang et al. 2012).

The morphological measures used in this work were obtained from the catalogue presented by Cassata et al. (2007), which provides information of non-parametric diagnostics of galaxy structure using the Hubble Space Telescope Advanced Camera for Surveys (ACS) for 232022 galaxies up to $\mathrm{F} 814 \mathrm{~W}=25$.

We cross-correlated the spatial positions of AGNs previously selected from Chang et al. catalogue with those presented by Cassata et al. (2007), using a matching radius of 1 arcsec. We find the following matches: 75 per cent and 85 per cent for obscured and unobscured AGNs and 85 per cent and 94 per cent for obscured and unobscured QSOs.

\footnotetext{
${ }^{2}$ We have used the Cutouts Service from the NASA/IPAC Infrared Science
} Archive http://irsa.ipac.caltech.edu/data/COSMOS/index_cutouts.html
In Fig. 8, we plot the M20 parameter versus Gini coefficient for the sample of obscured and unobscured (open and filled circles) AGNs (left panel) and for obscured and unobscured (open and filled squares) QSOs (right panel). The dashed line represent the criterion published by Lotz et al. (2008), according to the following definitions, Mergers: $G>-0.14 M_{20}+0.33$, Early(E/S0/Sa): $G<$ $-0.14 M_{20}+0.33$, and $G>0.14 M_{20}+0.80$, Late(Sb/Sc/Ir): $G<$ $-0.14 M_{20}+0.33$, and $G>0.14 M_{20}+0.80$.

We find that only 4 percent and 1 per cent of unobscured AGNs and QSOs, respectively are located in the region identified by mergers.

In the case of unobscured AGNs (filled circles, left panel), can be observed the presence of some objects in the area with low Gini indices $(G \lesssim 0.45)$. A visual inspection of them indicated that they are objects with irregular morphology.

Some works show a link between mergers and powerful obscured AGNs. Glikman et al. (2015) from a study using the Hubble Space Telescope (HST) Wide-Field Camera 3 (WFC3) near-infrared camera to image the host galaxies of a sample of 11 luminous, dustreddened QSOs at $z \sim 2$ reveal that 8/11 of these QSOs have actively merging hosts. Similar results were found by Assef et al. (2015) and Fan et al. (2016) in a sample of powerful, heavily obscured WISEselected QSOs at $\mathrm{z} \sim 2$, which show a large fraction of mergers in their host galaxies. On the contrary, other works do not show these trends. In a sample of X-ray selected AGN at $z \sim 2$, Kocevski et al. (2012) found that AGNs do not exhibit a significant excess of distorted morphologies while a large fraction reside in late-type galaxies. Schawinski et al. (2011) showed for a sample of X-ray selected AGNs with $1.5<z<3$ selected from the Chandra Deep Field South that the majority ( 80 per cent) of these AGNs have low Sérsic indices indicative of disk-dominated light profiles and undisturbed morphologies.

The lack of mergers signatures in our AGN and QSO samples observed in the M20 versus Gini plane suggests that either secular evolution, disk instability-driven accretion, minor mergers, or a combination of the three, play a major role in triggering AGN activity at these redshifts than previously thought (Schawinski et al. 2011).

\section{GALAXY DENSITY}

In this Section, we analyse the environment of obscured and unobscured AGNs and QSOs. According to the postulates of the unified model, the host galaxies of the different AGNs would exhibit similar properties and would be located in similar galactic environments. In Fig. 9 we show the projected radial density of tracer galaxies with $\left|z_{A G N}-z_{\text {galaxy }}\right| \leq 0.2$ and $r<25.75$ (which is the limiting apparent magnitude obtain from the Subaru $r$ band number counts) around the obscured and unobscured AGNs (left panel) and QSOs (right panel) with $r_{p}<500 \mathrm{kpc}$. We also include the sample of SF galaxies in this analysis as a comparison sample. Error bars were estimated using Poissonian errors. For the AGN sample we find that in small scales $(<100 \mathrm{kpc})$ unobscured objects show more neighbouring galaxies than the sample of obscured AGNs. While SF galaxies have a density of neighbouring galaxies similar to the sample of unobscured AGNs. In the case of the QSOs, we find that both obscured and unobscured samples have lower galaxy densities than that obtained for the SF galaxy sample. Despite uncertainties at smaller scales, unobscured QSOs also show, more neighbouring galaxies than the sample of obscured QSOs. We have also performed tests using other redshift limits, changing the redshift difference cut to $\left|z_{A G N}-z_{\text {galaxy }}\right| \leq 0.1$ and 0.15 does not change the results except that the number of tracer galaxies is reduced. 

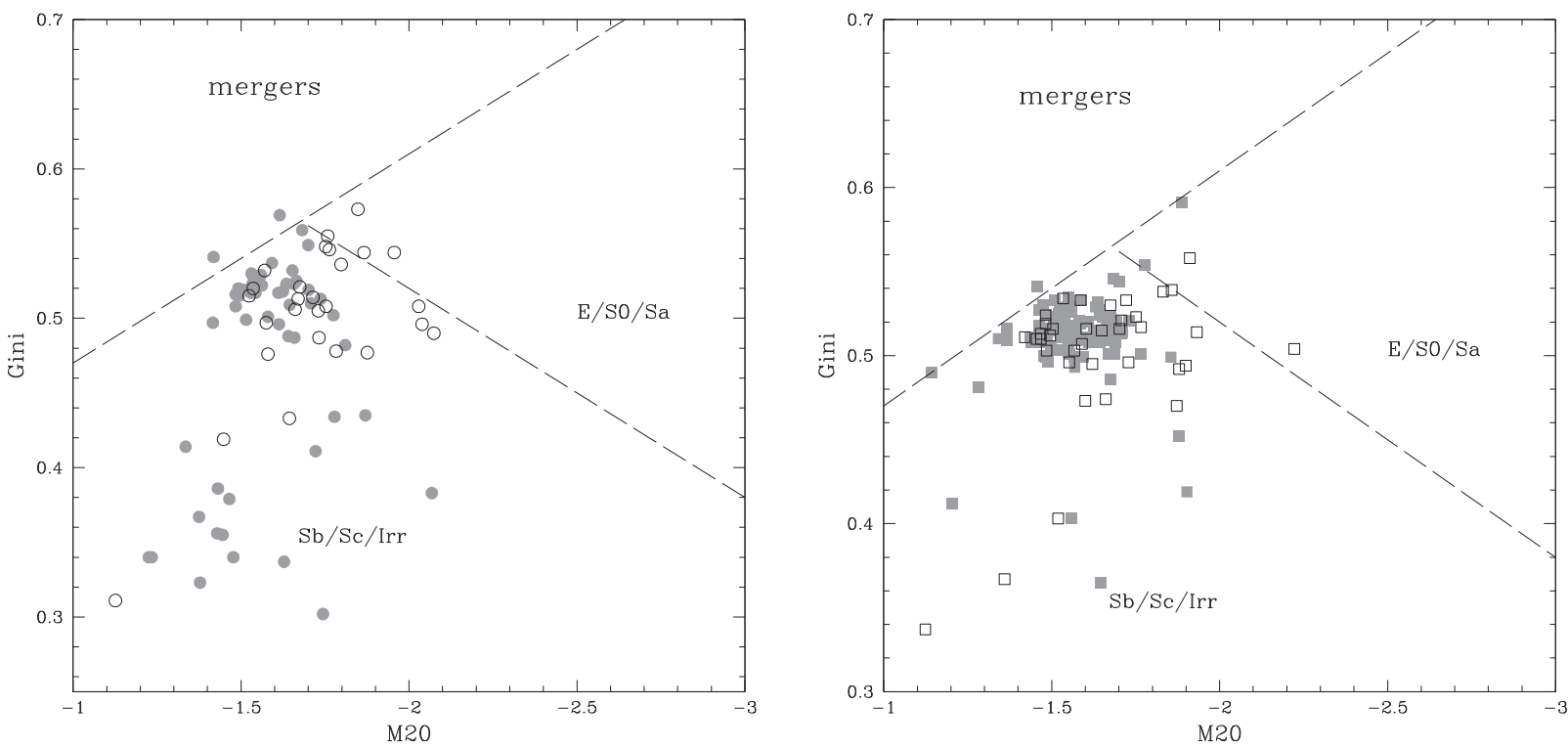

Figure 8. Gini versus M20 coefficient (G) for obscured and unobscured (open and filled circles, respectively) AGNs (left panel) and obscured and unobscured (open and filled squares, respectively) QSOs (right panel). Dividing lines show regions of mergers, Sb/Sc/Irr, and E/S0/Sa galaxy types taken from Lotz et al. (2008).
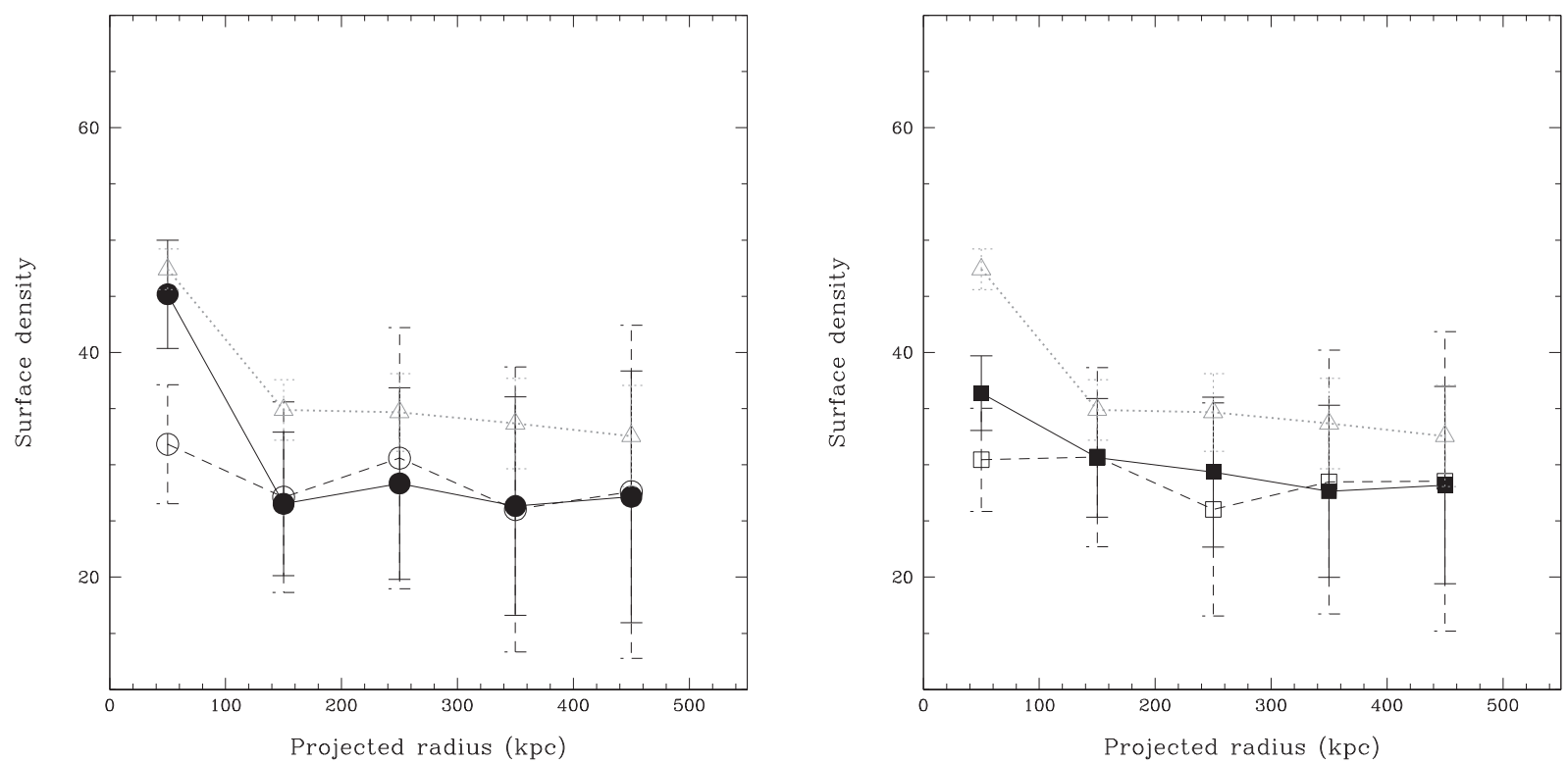

Figure 9. Projected radial density of tracer galaxies with $\left|z_{A G N}-z_{\text {galaxy }}\right| \leq 0.2$ and $r<25.75$ around the selected obscured and unobscured (open and filled circles) AGNs (left panel) and for obscured and unobscured (open and filled squares) QSOs (right panel). Open triangles in each figure represent the corresponding values for SF galaxies. The error bars represent the standard deviation within each data bin, estimated using Poissonian errors.

Environments of the different AGN samples show contradictory results at low and high redshifts in the literature. Allevato et al. (2011) analysed a sample of X-ray selected AGNs from the XMMCOSMOS survey with $z<1.5$ and found a difference in the environments between X-ray obscured and unobscured AGNs, who inhabit dark matter haloes with mass $\log \left(M_{\text {obs }}[\mathrm{M} \odot]\right)=13$ and $\log \left(M_{\text {unobs }}[\mathrm{M} \odot]\right)=13.3$. In a similar way, Allevato et al. (2014) studied a sample of AGNs selected on the basis of a combined Xray, optical spectra and photometric data from Chandra and XMM in the COSMOS area. These authors found that unobscured AGNs at $z \sim 3$ reside in 10 times more massive haloes compared to obscured AGNs. Gilli et al. (2009) analysed a sample of AGNs selected from the XMM-COSMOS survey with median redshifts $\bar{z} \sim 1$. These authors studied several samples of AGNs, among them a sample of X-ray selected AGNs using a criterion according to the hardness ratio, a subsample with $H R>-0.2$ (obscured) and other with $H R<-0.2$ (unobscured). The spatial clustering of these two sample shows that obscured and unobscured AGNs inhabit similar environments. Melnyk et al. (2018) studied the large (>1 Mpc) and small $(<0.4 \mathrm{Mpc})$ scale environment of X-ray selected AGNs with $z \sim 1$ in the XXL survey using the nearest neighbour distance method and the local galaxy density. No significant differences in the clustering of obscured $(\mathrm{HR}>-0.2)$ and unobscured $(\mathrm{HR}<$ $-0.2)$ AGNs were found. 

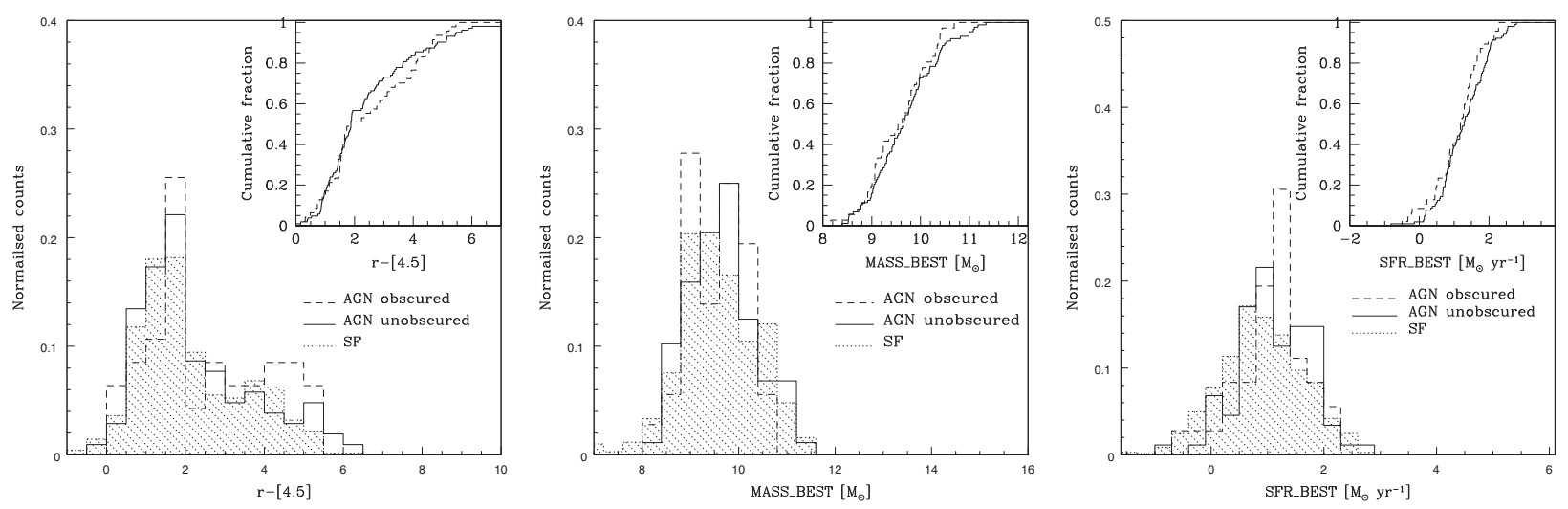

Figure 10. $r-(4.5)$ colour (left panel), stellar masses (middle panel), and SFR (right panel) distributions for tracer galaxies with $\left|z_{A G N}-z_{\text {galaxy }}\right| \leq 0.2$ and $r$ $<25.75$ within $100 \mathrm{kpc}$ from obscured (dashed line histogram) and unobscured (solid line histogram) AGNs. Shaded histograms represent the corresponding values for tracer galaxies in the field of SF galaxies. The inset shows the cumulative fraction distributions.

\section{NEIGHBOUR GALAXY PROPERTIES}

\subsection{Colour, stellar mass, and SFR of neighbouring galaxies}

It is well known that the properties of galaxies depend on the environment in which they reside. In this sense, one of the pioneering works on the effect of the environment on the properties of galaxies was that of Dressler (1980). This author studied the relation galaxy morphology-density in which, as the density of the environment increases, an increasing elliptical and S0 population is found, along with a corresponding decrease of those galaxies with a spiral morphology (Postman \& Geller 1984; Cappellari et al. 2011; Alpaslan et al. 2015). Other physical properties of galaxies that also depend on morphology, such as colour (Tanaka et al. 2004; Balogh et al. 2004), and SFR (Grützbauch et al. 2011). It also be expected that AGN properties are related on the environment, and it could be the environment that triggers processes, such as AGN accretion (Padilla, Lambas \& González 2010; Wang et al. 2017).

Here we study colours, masses, and SFRs of neighbour tracer galaxies located in the field of obscured and unobscured AGN and QSO samples. In Fig. 10, we show the $r-(4.5)$ colour (left panel), stellar masses (MASS_BEST, middle panel), and SFR (right panel) for tracer galaxies with $\left|z_{A G N}-z_{\text {galaxy }}\right| \leq 0.2$ and $r<25.75$ within $100 \mathrm{kpc}$ from obscured and unobscured AGN sample, which is the projected distance where we see differences in the environments. We have included the cumulative fraction distribution for the QSO and AGN samples (top right on each figure), in order to make clearer the differences between the different distributions. We have also included the corresponding distributions of neighbouring galaxies of the sample of SF galaxies (shaded histogram).

We find that the colour distribution of tracer galaxies in the field of obscured AGN presents an excess of galaxies with red colours, compared to the samples of unobscured AGN and the sample of SF galaxies. We have used the GMM code in order to quantify the colour distribution. For the sample of tracer galaxies in the field of obscured AGNs, we find $\mu_{1}=1.4 \pm 0.1$ and $\mu_{2}=4.0 \pm 0.3$, while for unobscured AGNs we obtained $\mu_{1}=1.5 \pm 0.2$ and $\mu_{2}=$ $4.0 \pm 0.6$. For the sample of tracer galaxies in the field of SF galaxies we find $\mu_{1}=1.40 \pm 0.05$ and $\mu_{2}=3.6 \pm 0.06$.

The stellar mass distribution of tracer galaxies in the field of obscured, unobscured AGNs and the SF galaxy sample are similar (see Fig. 10, middle panel). For the obscured and unobscured AGN sample we obtain $\overline{\log M_{o b s}}=9.5 \pm 0.6\left(\mathrm{M}_{\odot}\right)$ and $\overline{\log M_{\text {unobs }}}=9.6 \pm 0.7\left(\mathrm{M}_{\odot}\right)$, while for the SF galaxies we find $\overline{\log M_{s f}}=9.5 \pm 0.8\left(\mathrm{M}_{\odot}\right)$.

The SFR of tracer galaxies in the field of different AGNs and SF galaxies are slightly different. Comparing the values obtained for SF galaxies, tracer galaxies in the field of both obscured and unobscured AGNs have higher SFR values. For the obscured and unobscured AGN sample $\overline{\log \left(S F R_{\text {obs }}\right)}=1.0 \pm 0.6$ and $\overline{\log \left(S F R_{\text {unobs }}\right)}=1.1 \pm$ 0.6. While for the SF galaxies we find $\overline{\log \left(S F R_{s f}\right)}=0.9 \pm 1.0$.

In Fig. 11, we plot the same parameters as in Fig. 10 for a sample of tracer galaxies with $100<r_{p}<500 \mathrm{kpc}$ from the sample of AGNs. As it can be seen, the $r-(4.5)$, stellar mass and SFR of tracer galaxies in the field of obscured, unobscured, and SF galaxies are similar.

In Figs 12 and 13 we performed a similar study for tracer galaxies in the field of QSOs. In the left panel of Fig. 12, it can be seen that the $r-(4.5)$ colours of tracer galaxies in the field of obscured QSOs are redder in comparison with the unobscured and SF galaxy sample. Using the GMM code, we obtained $\mu_{1}=1.7 \pm 0.1$ and $\mu_{2}=$ $4.4 \pm 0.1$ for tracer galaxies in the field of obscured QSOs and $\mu_{1}=1.6 \pm 0.1$ and $\mu_{2}=4.4 \pm 0.3$ for the sample of unobscured QSOs.

The stellar mass and the SFR of tracer galaxies do not present large differences between the different QSO and SF galaxy samples. For the stellar mass distribution we find: $\mu_{o b s}=9.6 \pm 0.7$ and $\mu_{\text {unobs }}=9.7 \pm 0.7$, while for the SF galaxy sample we find $\mu_{s f}=9.5 \pm 0.8$. We find $\overline{\log \left(S F R_{o b s}\right)}=1.1 \pm 0.6\left(\mathrm{M}_{\odot} y r^{-1}\right)$ and $\overline{\log \left(S F R_{\text {unobs }}\right)}=0.9 \pm 0.6\left(\mathrm{M}_{\odot} y r^{-1}\right)$ for SFR of tracer galaxies. For the SF galaxies we obtain $\left.\overline{\log \left(S F R_{s f}\right.}\right)=0.9 \pm 1.0\left(\mathrm{M}_{\odot} y r^{-1}\right)$.

We find similar results to those observed in the AGN sample for colours, stellar masses, and SFRs of tracer galaxies with $100<r_{p}$ $<500 \mathrm{kpc}$ of the different AGN types.

\subsection{Morphology of neighbouring galaxies}

We analyse in this section the morphology of the neighbouring tracer galaxies in the field of different AGN and QSO types. We cross-matched our catalogue of tracer galaxies with $\left|z_{A G N}-z_{\text {galaxy }}\right|$ $\leq 0.2$ and $r<25.75$ in the field of AGN and QSO samples with the morphology catalogue presented by Cassata et al. (2007). We find that $\sim 40$ per cent of the tracers have a counterpart in the catalogue of Cassata et al. The morphological parameters in Cassata et al. catalogue are combined to classify galaxies defined through the parameter AUTO_CLASS as $1=$ early-type; $2=$ spiral; $3=$ 

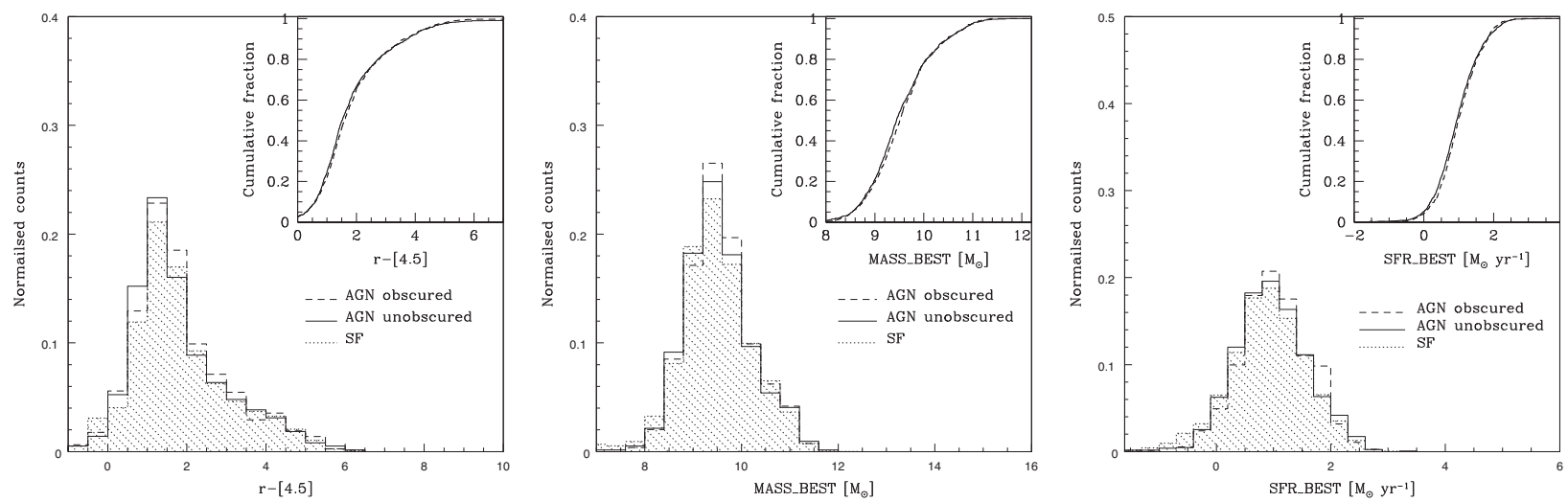

Figure 11. Same as Fig. 10 for a sample of tracer galaxies with $100<r_{p}<500 \mathrm{kpc}$ from the sample of AGNs.
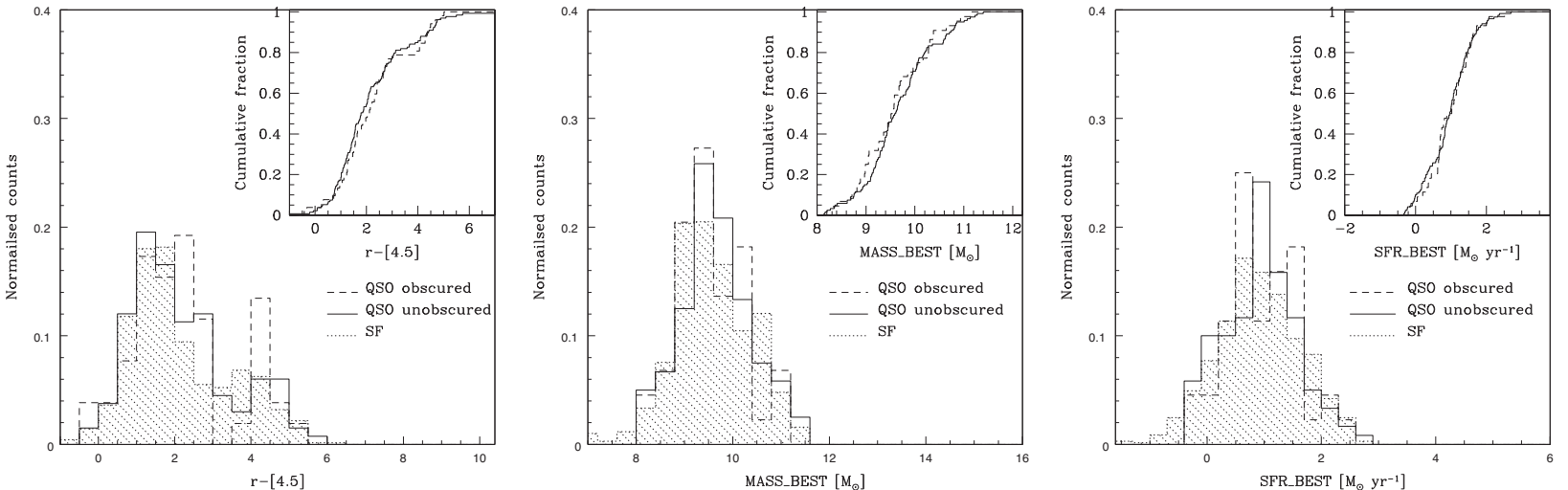

Figure 12. $r-(4.5)$ colour (left panel), stellar masses (middle panel), and SFR (right panel) distribution for tracer galaxies with $\left|z_{A G N}-z_{\text {galaxy }}\right| \leq 0.2$ and $r$ $<25.75$ within $100 \mathrm{kpc}$ from obscured (dashed line histogram) and unobscured (solid line histogram) QSOs. Shaded histograms represent the corresponding values for tracer galaxies in the field of SF galaxies. The inset shows the cumulative fraction distributions.
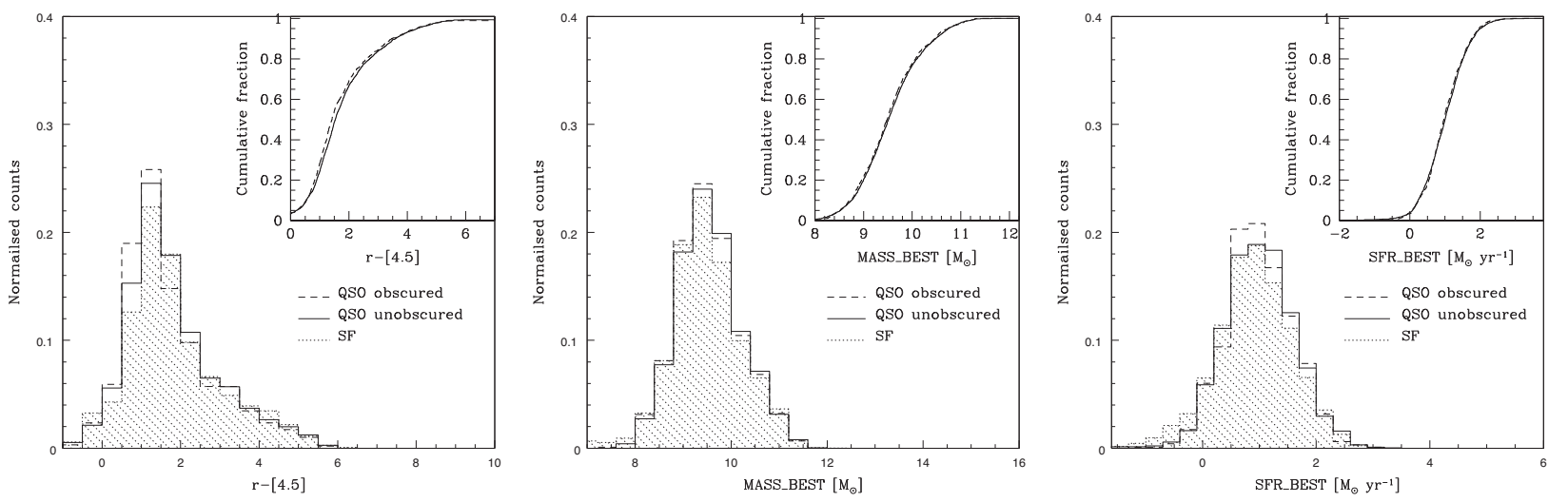

Figure 13. Same as Fig. 12 for a sample of tracer galaxies with $100<r_{p}<500 \mathrm{kpc}$ from the sample of QSOs.

irregular. We plot in Fig. 14 the corresponding AUTO_CLASS distribution in the field of obscured (dashed line histogram) and unobscured (solid line histogram) QSOs and AGNs. We included also the corresponding values for tracer galaxies in the field of SF galaxies (dotted lines). In all cases we divided the distribution for 3 different projected radius of $r_{p}<100 \mathrm{kpc}, 100<r_{p}<200 \mathrm{kpc}$, and for $r_{p}<500 \mathrm{kpc}$. At $r_{p}<100 \mathrm{kpc}$, obscured and unobscured AGNs have a higher percentage of spiral tracer galaxies compared to the results obtained in the field of QSOs. For the QSO sample, we find a lower percentage of irregular galaxies in the field of obscured QSOs with $r_{p}<100 \mathrm{kpc}$ (see Fig. 14, left panel). For projected radius between $100<r_{p}<200 \mathrm{kpc}$, we find that obscured AGNs have more irregular tracer galaxies compared to the sample of obscured QSOs and SF galaxies. At $r_{p}<500 \mathrm{kpc}$, both AGN, QSO, and SF galaxies have similar percentage of early, spiral and irregular tracer galaxies.

\section{SUMMARY AND DISCUSSION}

In this paper, we have studied the properties of host galaxies and their environment for a sample of obscured and unobscured X-ray 

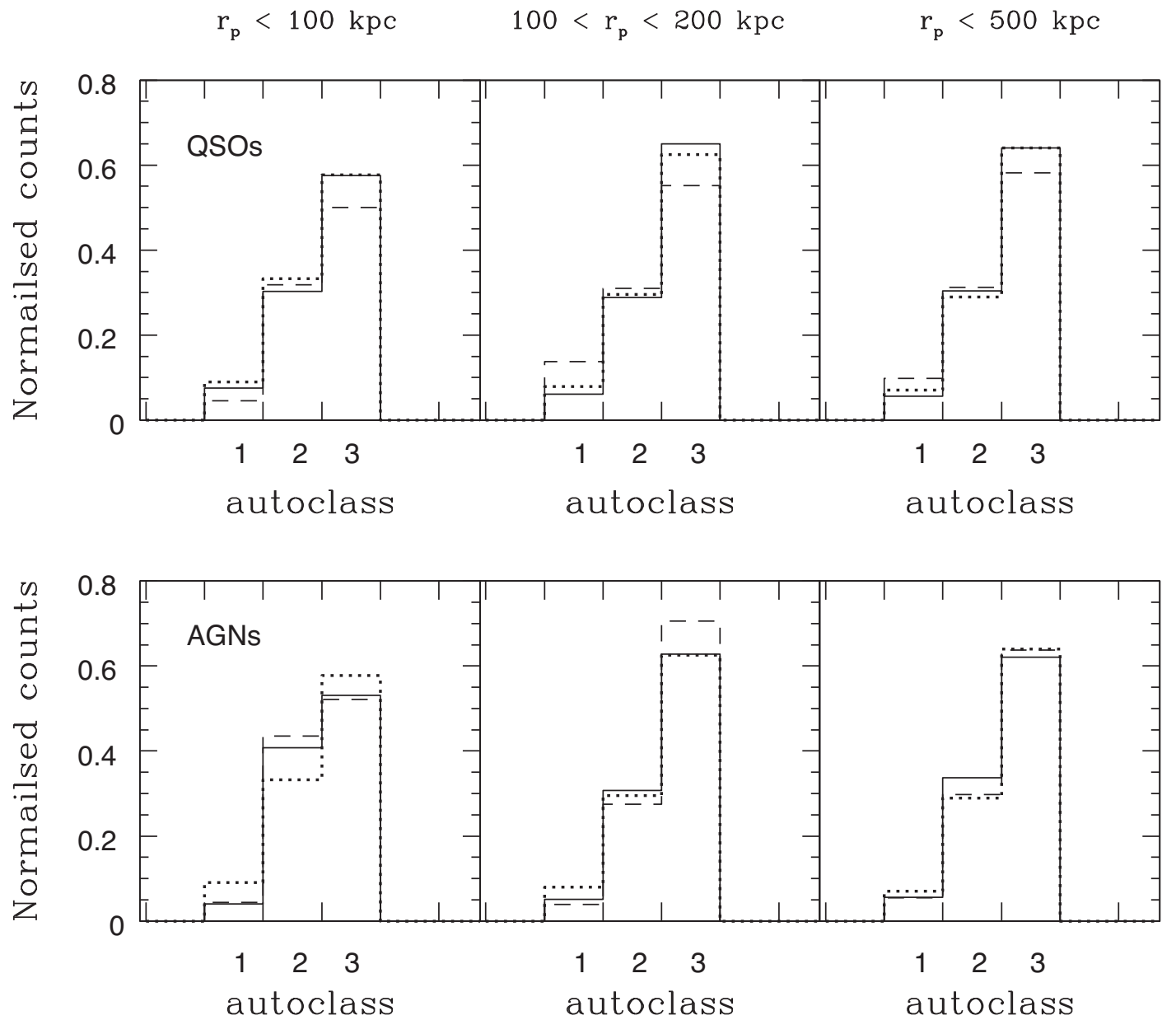

Figure 14. Morphology estimator AUTO_CLASS distribution for tracer galaxies with $\left|z_{A G N}-z_{\text {galaxy }}\right| \leq 0.2$ and $r<25.75$ in the field of obscured (dashed lines) and unobscured (solid lines) QSOs (upper panel) and AGNs (lower panel). Dotted lines represent the corresponding values for tracer galaxies in the field of SF galaxies. AUTO_CLASS values correspond to $1=$ early-type; $2=$ spiral; $3=$ irregular.

selected AGNs and QSOs at high redshifts $(1.4 \leq z \leq 2.5)$ selected from Chang et al. catalogue in the COSMOS survey area.

The main results can be summarized as follows:

(i) The optical-IR colour, $r-(4.5)$ of obscured and unobscured AGNs is bimodal. The colour separation threshold is consistent with the value found by Hickox et al. (2011) and Bornancini et al. (2017), $r-(4.5) \sim 3$. The sample of obscured AGNs is divided into two parts: one consisting of host galaxies with predominantly red colours, and a small population with blue colours. In the case of obscured QSOs, we find also that the colour distribution is bimodal, consisting in two similar population of objects with red and blue colours, while unobscured QSOs have predominantly blue hosts.

(ii) The rest-frame colours of obscured and unobscured AGNs and QSOs observed in the $M_{N U V}-M_{r}$ versus $M_{r}-M_{J}$ colour diagram show that most objects are located in the area defined by starforming galaxies, rather than quiescent galaxies. We also find bimodalities in $M_{N U V}-M_{r}$ colour distribution in unobscured AGN and QSO samples as well as in obscured QSOs.

(iii) The stellar mass versus size relation for AGN and QSO hosts shows that in general, these objects are more compact and massive in comparison to a control sample SF galaxies. The stellar mass distribution of both AGN and QSO hosts are consistent with massive galaxies compared to the sample of SF galaxies. The stellar mass distribution of obscured and unobscured AGNs are similar, while unobscured QSOs are found more massive than obscured ones by $\Delta \overline{\log M}=0.3 \operatorname{dex}\left(\mathrm{M}_{\odot}\right)$.

(iv) From a morphology analysis using non-parametric measures, such as the Gini coefficient and the M20 parameter, we obtained that most of the AGNs and QSOs are located in regions belonging to late-type galaxies or irregulars. Only a small percentage are related to early-type galaxies and mergers.

(v) The projected radial density of tracer galaxies around the sample of AGNs and QSOs show that both obscured and unobscured AGNs and QSOs inhabit similar galaxy environment. Only at small scales $(<100 \mathrm{kpc})$ unobscured AGNs show more neighbouring galaxies than the sample of obscured AGNs. Unobscured AGNs show a similar radial profile as the sample of SF galaxies. For QSOs we find that both obscured and unobscured samples have lower galaxy densities than that obtained for the SF sample. Despite errors at smaller scales, unobscured QSOs show also, more neighbouring galaxies than the sample of obscured QSOs. Our results are consistent with those previously obtained by Allevato et al. (2014) in the sense that Type I AGNs reside in high-density regions at high redshifts, which may have evolved earlier. This contrasts with the findings of Melnyk et al. (2018), who obtained similar environment for the different AGN types.

(vi) We find that the colour distribution of tracer galaxies with $r_{p}$ $<100 \mathrm{kpc}$ around the field of obscured AGN presents an excess of galaxies with red colours, compared to the samples of unobscured 
AGN and the sample of SF galaxies. The stellar mass distribution of tracer galaxies with $r_{p}<100 \mathrm{kpc}$ in the field of obscured, unobscured AGNs and SF galaxy sample are similar. The SFR of tracer galaxies with $r_{p}<100 \mathrm{kpc}$ around obscured and unobscured AGNs present similar distributions and have values larger than the SFR of tracer galaxies in the surroundings of SF galaxies.

For the case of QSOs, the stellar mass and the SFR of tracer galaxies do not present large differences.

(vii) From an analysis of the morphology of tracer galaxies in the field of different AGN types we find that obscured and unobscured AGNs have a higher percentage of spiral tracer galaxies compared to the results obtained in the surroundings of both obscured and unobscured QSOs at within $r_{p}<100 \mathrm{kpc}$. In the case of QSOs, we find a lower percentage of irregular galaxies in the field of obscured QSOs with $r_{p}<100 \mathrm{kpc}$. Unobscured QSOs and SF galaxies present equal number of irregular tracer galaxies.

The differences of host galaxy properties, such as UV/optical/IR colours and masses, together with the differences found in the projected galaxy density at small scales $\left(r_{p}<100 \mathrm{kpc}\right)$ and neighbouring galaxy properties, favour an evolutionary scenario rather than a strict unified model.

Although the unified model may be valid in some cases (for example at low redshifts), large-scale evolutionary processes undergone by a galaxy can cause its nucleus to become either obscured or unobscured during its lifetime. Since the AGN existence is short ( $\lesssim 0.001$ Gigayear, Schawinski et al. 2015) compared to the merger stage ( $\lesssim 1$ Gigayear, Conselice 2006), possibly an important part of the galaxies repeatedly experienced AGN activity throughout its history due to gas infall to the central engine by disc instabilities or secular processes. Since, the objects studied in this work are at high redshifts and the X-ray luminosity of these AGNs is 10-100 times greater than those at $z<1$, unobscured AGN or QSO phase might probably correspond to the end of an obscured phase where the growing BHs can produce highly enough accretion luminosity to sweep the surrounding material.

\section{ACKNOWLEDGEMENTS}

We sincerely thank the anonymous referee for useful suggestions, which improved the the quality of this manuscript. Based on data products from observations made with ESO Telescopes at the La Silla Paranal Observatory under ESO programme ID 179.A-2005 and on data products produced by TERAPIX and the Cambridge Astronomy Survey Unit on behalf of the UltraVISTA consortium. Based on data obtained with the European Southern Observatory Very Large Telescope, Paranal, Chile, under Large Programs 175.A-0839 (zCOSMOS), 179.A-2005 (UltraVista), and 185.A0791 (VUDS). This work was partially supported by the Consejo Nacional de Investigaciones Científicas y Técnicas (CONICET) and the Secretaría de Ciencia y Tecnología de la Universidad de Córdoba (SeCyT).

\section{REFERENCES}

Abraham R. G., van den Bergh S., Nair P., 2003, ApJ, 588, 218

Allevato V. et al., 2011, ApJ, 736, 99

Allevato V. et al., 2014, ApJ, 796, 4

Alonso-Herrero A. et al., 2011, ApJ, 736, 82

Alpaslan M. et al., 2015, MNRAS, 451, 3249

Antonucci R., 1993, ARA\&A, 31, 473

Arnouts S. et al., 2013, A\&A, 558, A67

Ashby M. L. N. et al., 2013, ApJ, 769, 80
Ashby M. L. N. et al., 2015, ApJS, 218, 33

Assef R. J. et al., 2015, ApJ, 804, 27

Audibert A., Riffel R., Sales D. A., Pastoriza M. G., Ruschel-Dutra D., 2017, MNRAS, 464, 2139

Baldry I. K. et al., 2004, ApJ, 600, 681

Balogh M. et al., 2004, MNRAS, 348, 1355

Barro G. et al., 2013, ApJ, 765, 104

Barro G. et al., 2014, ApJ, 795, 145

Beifiori A., Courteau S., Corsini E. M., Zhu Y., 2012, MNRAS, 419, 2497

Bennert V. N. et al., 2015, ApJ, 809, 20

Best P. N., von der Linden A., Kauffmann G., Heckman T. M., Kaiser C. R., 2007, MNRAS, 379, 894

Bongiorno A. et al., 2012, MNRAS, 427, 3103

Bornancini C., García Lambas D., 2018, MNRAS, 479, 2308

Bornancini C. G., O’Mill A. L., Gurovich S., Lambas D. G., 2010, MNRAS, 406, 197

Bornancini C. G., Taormina M. S., Lambas D. G., 2017, A\&A, 605, A10

Caplar N., Lilly S. J., Trakhtenbrot B., 2015, ApJ, 811, 148

Caplar N., Lilly S. J., Trakhtenbrot B., 2018, ApJ, 867, 148

Cappellari M. et al., 2011, MNRAS, 416, 1680

Cappelluti N. et al., 2009, A\&A, 497, 635

Cassata P. et al., 2007, ApJS, 172, 270

Cattaneo A., Blaizot J., Devriendt J., Guiderdoni B., 2005, MNRAS, 364, 407

Chang Y.-Y. et al., 2017, ApJS, 233, 19

Civano F. et al., 2015, ApJ, 808, 185

Civano F. et al., 2016, ApJ, 819, 62

Conselice C. J., 2006, ApJ, 638, 686

D’Onofrio M., Marziani P., Sulentic J. W., 2012, Fifty Years of Quasars: From Early Observations and Ideas to Future Research, Astrophysics and Space Science Library, Volume 386, Springer-Verlag, Berlin Heidelberg

Daddi E. et al., 2004, ApJ, 617, 746

Daddi E. et al., 2005, ApJ, 626, 680

Damjanov I. et al., 2009, ApJ, 695, 101

Di Matteo T., Springel V., Hernquist L., 2005, Nature, 433, 604

DiPompeo M. A., Myers A. D., Hickox R. C., Geach J. E., Hainline K. N., 2014, MNRAS, 442, 3443

Donley J. L., Rieke G. H., Pérez-González P. G., Barro G., 2008, ApJ, 687, 111

Donley J. L. et al., 2012, ApJ, 748, 142

Donoso E., Yan L., Stern D., Assef R. J., 2014, ApJ, 789, 44

Dressler A., 1980, ApJ, 236, 351

Elitzur M., Shlosman I., 2006, ApJ, 648, L101

Elvis M. et al., 2009, ApJS, 184, 158

Fan L. et al., 2016, ApJ, 822, L32

Ferrarese L., Merritt D., 2000, ApJ, 539, L9

Förster Schreiber N. M. et al., 2009, ApJ, 706, 1364

Gebhardt K. et al., 2000, ApJ, 539, L13

Gilli R. et al., 2009, A\&A, 494, 33

Glikman E., Simmons B., Mailly M., Schawinski K., Urry C. M., Lacy M., 2015, ApJ, 806, 218

Goulding A. D., Alexander D. M., 2009, MNRAS, 398, 1165.

Goulding A. D. et al., 2012, ApJ, 755, 5

Graham A. W., Onken C. A., Athanassoula E., Combes F., 2011, MNRAS, 412, 2211

Greene J. E., Ho L. C., Barth A. J., 2008, ApJ, 688, 159

Grützbauch R. et al., 2011, MNRAS, 411, 929

Guainazzi M. et al., 2001, MNRAS, 327, 323.

Hasinger G., et al., 2007, ApJS, 172, 29

Hatch N. A. et al., 2011, MNRAS, 410, 1537

Hickox R. C., Alexander D. M., 2018, ARA\&A, 56, 625

Hickox R. C. et al., 2007, ApJ, 671, 1365

Hickox R. C. et al., 2009, ApJ, 696, 891

Hickox R. C. et al., 2011, ApJ, 731, 117

Hopkins P. F. et al., 2006, ApJS, 163, 1

Hopkins P. F., Robertson B., Krause E., Hernquist L., Cox T. J., 2006, ApJ, 652, 107

Hopkins P. F., Hernquist L., Cox T. J., Kereš D., 2008, ApJS, 175, 356 
Ilbert O. et al., 2010, ApJ, 709, 644

Ilbert O. et al., 2013, A\&A, 556, A55

Jiang N. et al., 2016, ApJ, 832, 111

Kauffmann G. et al., 2003, MNRAS, 341, 54

Kauffmann G. et al., 2007, ApJS, 173, 357

Kembhavi A. K., Narlikar J. V., 1999, in Kembhavi A. K., Narlikar J. V., eds, Quasars and Active Galactic Nuclei: An Introduction. Cambridge Univ. Press, Cambridge, UK

Kocevski D. D. et al., 2012, ApJ, 744, 148

Kong X. et al., 2006, ApJ, 638, 72

Kong X., Fang G., Arimoto N., Wang M., 2009, ApJ, 702, 1458

Kormendy J., 1988a, ApJ, 325, 128

Kormendy J., 1988b, ApJ, 335, 40

Kormendy J., Ho L. C., 2013, ARA\&A, 51, 511

Kriek M., van Dokkum P. G., Labbé I., Franx M., Illingworth G. D., Marchesini D., Quadri R. F., 2009, ApJ, 700, 221

Lacy M. et al., 2004, ApJS, 154, 166

Laigle C. et al., 2016, ApJS, 224, 24

Le Floc'h E. et al., 2009, ApJ, 703, 222

Lehmer B. D. et al., 2012, ApJ, 752, 46

Lin L. et al., 2016, ApJ, 817, 97

Lotz J. M., Primack J., Madau P., 2004, AJ, 128, 163

Lotz J. M. et al., 2008, ApJ, 672, 177-197

Ly C. et al., 2009, ApJ, 697, 1410

Lynden-Bell D., 1969, Nature, 223, 690

Magorrian J. et al., 1998, AJ, 115, 2285

Malkan M. A., Gorjian V., Tam R., 1998, ApJS, 117, 25

Marchesi S. et al., 2016, ApJ, 817, 34

Marconi A., Hunt L. K., 2003, ApJ, 589, L21

Matt G., 2000, A\&A, 355, L31.

McCracken H. J. et al., 2010, ApJ, 708, 202

McCracken H. J. et al., 2012, A\&A, 544, A156

Melnyk O. et al., 2018, A\&A, 620, A6

Miley G., De Breuck C., 2008, A\&A Rev., 15, 67

Miley G. K. et al., 2004, Nature, 427, 47

Miley G. K. et al., 2006, ApJ, 650, L29

Moutard T. et al., 2016, A\&A, 590, A103

Muratov A. L., Gnedin O. Y., 2010, ApJ, 718, 1266

Nenkova M., Ivezić Ž., Elitzur M., 2002, ApJ, 570, L9

Netzer H., 2015, ARA\&A, 53, 365

Oke J. B., Gunn J. E., 1983, ApJ, 266, 713

Overzier R. A., 2016, A\&A Rev., 24, 14

Padilla N., Lambas D. G., González R., 2010, MNRAS, 409, 936

Patel S. G., Kelson D. D., Holden B. P., Franx M., Illingworth G. D., 2011, ApJ, 735, 53

Pierce C. M. et al., 2010, MNRAS, 408, 139

Postman M., Geller M. J., 1984, ApJ, 281, 95

Ramos Almeida C. et al., 2011, ApJ, 731, 92

Rees M. J., 1984, ARA\&A, 22, 471

Reunanen J., Kotilainen J. K., Prieto M. A., 2003, MNRAS, 343, 192

Saglia R. P. et al., 2016, ApJ, 818, 47

Salpeter E. E., 1964, ApJ, 140, 796
Sanders D. B. et al., 1988, ApJ, 325, 74

Sanders D. B. et al., 2007, ApJS, 172, 86

Sanghvi J. et al., 2014, MNRAS, 445, 1261

Sani E., Marconi A., Hunt L. K., Risaliti G., 2011, MNRAS, 413, 1479

Savorgnan G. A. D., Graham A. W., Marconi A., Sani E., 2016, ApJ, 817, 21

Schawinski K. et al., 2011, ApJ, 727, L31

Schawinski K., Koss M., Berney S., Sartori L. F., 2015, MNRAS, 451, 2517

Schmidt M., 1963, Nature, 197, 1040

Schulze A., Wisotzki L., 2011, A\&A, 535, A87

Scoville N. et al., 2007, ApJS, 172, 1

Shakura N. I., Sunyaev R. A., 1973, A\&A, 24, 337

Shankar F. et al., 2019, MNRAS, 485, 1278

Shen J., Vanden Berk D. E., Schneider D. P., Hall P. B., 2008, AJ, 135, 928

Shen Y. et al., 2015, ApJ, 805, 96

Shields G. A. et al., 2003, ApJ, 583, 124

Shimakawa R. et al., 2018, MNRAS, 481, 5630

Springel V., Di Matteo T., Hernquist L., 2005a, MNRAS, 361, 776

Springel V. et al., 2005b, Nature, 435, 629

Stern D. et al., 2012, ApJ, 753, 30

Szomoru D., Franx M., Bouwens R. J., van Dokkum P. G., Labbé I., Illingworth G. D., Trenti M., 2011, ApJ, 735, L22

Tanaka M., Goto T., Okamura S., Shimasaku K., Brinkmann J., 2004, AJ, 128,2677

Taniguchi Y. et al., 2007, ApJS, 172, 9

Taniguchi Y. et al., 2015, PASJ, 67, 104

Toft S. et al., 2007, ApJ, 671, 285

Treister E. et al., 2009, ApJ, 693, 1713

Tremaine S. et al., 2002, ApJ, 574, 740

Treu T., Woo J.-H., Malkan M. A., Blandford R. D., 2007, ApJ, 667, 117

Trujillo I., Conselice C. J., Bundy K., Cooper M. C., Eisenhardt P., Ellis R. S., 2007, MNRAS, 382, 109

Urry C. M., Padovani P., 1995, PASP, 107, 803

Venemans B. P. et al., 2002, ApJ, 569, L11

Volonteri M., Haardt F., Madau P., 2003, ApJ, 582, 559

Wang T. et al., 2012, ApJ, 752, 134

Wang T. et al., 2017, A\&A, 601, A63

Williams R. J., Quadri R. F., Franx M., van Dokkum P., Labbé I., 2009, ApJ, 691,1879

Williams R. J., Quadri R. F., Franx M., van Dokkum P., Toft S., Kriek M., Labbé I., 2010, ApJ, 713, 738

Woo J.-H., Treu T., Malkan M. A., Blandford R. D., 2006, ApJ, 645, 900

Wuyts S. et al., 2011, ApJ, 738, 106

Xu D. et al., 2012, AJ, 143, 83

Yang G. et al., 2019, MNRAS, 485, 3721

Zamojski M. A. et al., 2007, ApJS, 172, 468

Zel'dovich Y. B., Novikov I. D., 1964, Soviet Physics Doklady, 9, 246

Zou F., Yang G., Brandt W. N., Xue Y., 2019, ApJ, 878, 11

This paper has been typeset from a $\mathrm{T}_{\mathrm{E}} \mathrm{X} / \mathrm{L} \mathrm{T}_{\mathrm{E}} \mathrm{X}$ file prepared by the author. 